\title{
THE GEOMETRY OF HYPERBOLIC AND ELLIPTIC CR-MANIFOLDS OF CODIMENSION TWO*
}

\author{
GERD SCHMALZ ${ }^{\dagger}$ AND JAN SLOVÁK ${ }^{\ddagger}$
}

\begin{abstract}
The general theory of parabolic geometries is applied to the study of the normal Cartan connections for all hyperbolic and elliptic 6-dimensional CR-manifolds of codimension two. These structures present a very distinguished case in CR-geometry with many features of the nondegenerate real hypersurfaces. The geometric meaning of the individual components of the torsion is explained and the chains of dimensions one and two are discussed.
\end{abstract}

There have been many attempts to use some ideas going back up to Cartan, in order to understand the geometry of CR-manifolds. In the codimension one cases, the satisfactory solution had been worked out in the seventies, see $[22,23,8]$, but the higher codimensions have not been understood yet in a comparable extent. In general, there are no canonical homogeneous models for higher codimensional CRstructures (since there is a continuum of non-isomorphic ones) and, furthermore, the spaces of automorphisms of these homogeneous models are much smaller than in the hypersurface case. There is an exception, however: the 6-dimensional CR-manifolds of codimension two. There are just three non-isomorphic non-degenerate homogeneous models for these geometries and the automorphism groups happen to be semi-simple for two of them. Compared to all other cases, not much has been known about these distinguished geometries, see [9] for more historical remarks and a few results.

In this paper, the recent general theory of the so called parabolic geometries is applied to the latter semi-simple cases. In particular, we use the approach developed in $[4,21]$, see also $[24,27]$ for earlier results. Relying on recent achievements by the authors, a clean and quite simple construction of the normal Cartan connection is presented. This Cartan connection replaces the absolute parallelisms from [9] by more powerful geometric tools and it enables the detailed study of geometrical and analytical properties of the CR structures. Consequently the resulting geometric picture is much more transparent and surprising new results are obtained.

The main advantage of our approach is the fully coordinate-free handling of the normal Cartan connection and its curvature. Thus we are able to translate the cohomological properties of the structure algebras into full geometrical understanding of the curvature obstruction, without writing down the curvature components explicitly. The initial section introduces the CR structures and provides a brief exposition of distinguished second order osculations of the surfaces by quadrics. Then we observe, that this osculation transfers enough data from the quadric to apply the general construction of normal Cartan connections, due to [24,4]. This leads easily to the main Theorems 1.2 and 1.3. In fact, the Cartan connections are constructed also for certain abstract CR-manifolds though the embedded ones have many distinguished properties. The second section is devoted to the exposition of the generalities on parabolic geometries modeled over $|2|$-graded algebras and provides the proof of the existence of the normal connections.

Next we study the local geometry of the hyperbolic points in detail. We recover easily all known facts from [9], but we go much further. In particular, we identify

\footnotetext{
*Received August 11, 1999; accepted for publication September 9, 1999.

$\dagger^{\dagger}$ Mathematisches Institut der Universität Bonn, Beringstraße 1, D-53115 Bonn, Germany.

$\ddagger$ Department of Algebra and Geometry, Masaryk University, Janackovo n. 2a, 66295 Brno, Czech Republic (slovak@math.muni.cz).
} 
the complete geometric obstructions against the integrability of the almost product structure on the tangent bundle (Theorem 3.5), the integrability of the almost complex structure on the tangent CR space (Theorem 3.6), and the compatibility of the almost product and almost complex structures (3.8). It turns out that the latter two obstructions always vanish on the embedded hyperbolic CR-structures which results in automatic vanishing of several algebraic brackets. In particular, the whole hyperbolic CR-manifold $M \subset \mathbb{C}^{4}$ is a product of two 3-dimensional CR-manifolds if an only if its almost product structure is integrable, see Theorem 3.9. Finally we discuss the chains of dimensions one and two.

Following our intuition, the geometric properties at hyperbolic points have been expected to have their counterparts in the local geometry at the elliptic points, $\mathrm{cf}$. remarks and open problems in [9]. This is the subject of Section 4. In particular, we observe that the roles of almost complex and almost product structures are swapped. Thus, there is an almost complex structure on the whole tangent bundle $T M$ and we distinguish the algebraic brackets obstructing its integrability in Theorem 4.3. The obstructions against the integrability of the almost product structures on the complex spaces $T^{C R} M$ and their compatibility with the almost complex structures vanish automatically for the embedded elliptic CR-structures. They are discussed in 4.4, 4.5. The analogy to the product property of torsion-free hyperbolic geometries is the holomorphic normal Cartan connection in the elliptic case, see Theorem 4.6. Finally we prove that for torsion-free elliptic geometries, there are unique one-dimensional complex chains in all complex directions transversal to the complex subbundle $T^{C R} M$ (Theorem 4.7).

The last section collects some conclusions and remarks on future applications. The necessary cohomologies are computed in Appendix A while some more details on the normalized osculations and the discussion of chains on the hyperbolic and elliptic quadrics is postponed to Appendix B.

The whole paper stresses the differential-geometric properties and we have confined the analytical problems and consequences to a few remarks. The function theoretical aspects will be discussed elsewhere.

ACKNOWLEDGEMENTS. The authors should like to mention very helpful discussions with Andreas Čap and Vladimír Souček. The research originates in discussions during the stay of both authors at University of Adelaide. Further support by SFB 256 at Universität Bonn, Masaryk University in Brno, and GAČR Grant Nr. 201/96/0079 has been important. Essential part of the writing was undertaken during the stay of the second author at the Max Planck Institute for Mathematics in Sciences in Leipzig.

1. CR-structures of codimension two. Let $M$ be a real submanifold in the complex space $\mathbb{C}^{N}$. Then there is the CR-subbundle $T^{\mathrm{CR}} M=T M \cap J(T M)$ which consists of all vectors $\xi_{x} \in T_{x} M$ such that the canonical complex structure $J$ on $\mathbb{C}^{N}$ maps $\xi_{x}$ to $J\left(\xi_{x}\right) \in T_{x} M$. We say that the $C R$-codimension of $M$ is $k$ if $\operatorname{dim} M$ is $2 n+k$ and $\operatorname{dim} T^{\mathrm{CR}} M$ is $2 n$. By means of the implicit function theorem, we may use a holomorphic projection of $\mathbb{C}^{N} \rightarrow \mathbb{C}^{n+k}$ and express $M$ locally as

$$
\operatorname{Im} w_{\nu}=f_{\nu}(z, \bar{z}, \operatorname{Re} w), \quad \nu=1, \ldots, k
$$

where $z=\left(z_{1}, \ldots, z_{n}\right), w=\left(w_{1}=u_{1}+i v_{1}, \ldots, w_{k}=u_{k}+i v_{k}\right)$ are coordinates in $\mathbb{C}^{n+k}$ and $f(0)=0, d f(0)=0$. Geometrically this means that the origin belongs to $M$ and $T_{0} M$ is just $\{v=0\}$. By means of further biholomorphic transformation of second order we are able to eliminate the "harmonic" part of the second order term 
in $f$ :

$$
\operatorname{Re} \sum \frac{\partial^{2} f}{\partial z_{i} \partial z_{j} \mid 0} z_{i} z_{j}+2 \operatorname{Re} \sum \frac{\partial^{2} f}{\partial z_{i} \partial u_{j} \mid 0} z_{i} u_{j}+\frac{1}{2} \sum \frac{\partial^{2} f}{\partial u_{i} \partial u_{j} \mid 0} u_{i} u_{j}
$$

Only the hermitian part in the second order term of $f$ :

$$
h(z, \bar{z})=\frac{1}{2} \sum{\frac{\partial^{2} f}{\partial z_{i} \partial \bar{z}_{j} \mid 0}}_{i} \bar{z}_{j}
$$

will remain, thus we achieve that $M$ is given by

$$
v=h(z, \bar{z})+\mathrm{O}(3)
$$

at a neighborhood of the origin. For more details see [19]. The vector-valued hermitian form $h$ shall be denoted by $\langle z, z\rangle$ in the sequel. The submanifold $M$ is called Levi nondegenerate (at the origin) if the scalar components of $\langle z, z\rangle$ are linearly independent and do not have a common anihilator. The Levi form $\frac{1}{2 i} h$ is given by means of the standard Lie bracket $\{,\}_{\text {Lie }}$ of vector fields modulo the complex subspace $T^{C R} M$, $\xi \mapsto\left\{\xi_{x}, J \xi_{x}\right\}_{\text {Lie }} \in T M / T^{\mathrm{CR}} M$ for the CR-vector fields $\xi, J \xi: M \rightarrow T^{\mathrm{CR}} M$. The latter bracket is algebraic since the standard Lie bracket composed with the projection onto the quotient is clearly linear over functions.

The geometric meaning of (1.1) is that $M$ osculates the quadric

$$
Q: v=\langle z, z\rangle
$$

in second order. Both $M$ and $Q$ share the same tangent space, CR-tangent space and Levi form at the origin.

Now, let us assume that $M \subset \mathbb{C}^{4}$ is of $\mathrm{CR}$-codimension 2 and assume further that $M$ is Levi non-degenerate. Thus $M$ is a smooth real 6-dimensional manifold.

The quadric $Q$ can be always understood as an open domain in the homogeneous space $G / P$ where $G$ is the group of the automorphisms of the quadric $Q$ and $P$ its isotropic subgroup of the origin. This means that the tangent space in the origin carries the $P$-module structure of $\mathfrak{g} / \mathfrak{p}$ in a canonical way and the second order data that are $P$-invariant can be carried over to $M$ from $\mathfrak{g} / \mathfrak{p}$ to the individual tangent spaces of $M$ by means of the osculation.

Thus, in order to try to study the geometry of $M$ in the spirit of the general theory. as briefly reviewed in Section 2, we have to distinguish the possible non-degenerate $\mathbb{C}^{2}$-valued hermitian forms by a suitable normalization and to analyze the remaining freedom in the osculation. This has been done in $[16,19]$, see Appendix B for a review. In particular, we can achieve one of the following three forms for $h(z, \bar{z})=\langle z, z\rangle \in \mathbb{C}^{2}$ by a linear transformation in $z$ 's and $v$ 's

$$
\begin{array}{ll}
h^{1}(z, \bar{z})=z_{1} \bar{z}_{1}, & h^{2}(z, \bar{z})=z_{2} \bar{z}_{2} \\
h^{1}(z, \bar{z})=z_{1} \bar{z}_{1}, & h^{2}(z, \bar{z})=\operatorname{Re} z_{1} \bar{z}_{2} \\
h^{1}(z, \bar{z})=\operatorname{Re} z_{1} \bar{z}_{2}, & h^{2}(z, \bar{z})=\operatorname{Im} z_{1} \bar{z}_{2}
\end{array}
$$

and we refer to these cases as to hyperbolic, parabolic, and elliptic, respectively. The normalization (1.1) with one of these hermitian forms $h$ is given uniquely up to the isotropic subgroup of the origin in the group of all biholomorphic automorphisms of $Q \subset \mathbb{C}^{4}$. 
We say that a point $x \in M$ is hyperbolic or parabolic or elliptic if the osculating quadric at $x$ is of that type. The set of all hyperbolic points is open and the same for the elliptic ones. The CR-structure on $M$ is called hyperbolic, or parabolic, or elliptic, if all points of $M$ are of the same type.

Let $M \subset \mathbb{C}^{4}$ be a CR-structure of codimension two, such that all its points are either hyperbolic or elliptic. As discussed above, the choice of the canonical form of the osculating quadric $Q=G / P$ reduces the freedom in the osculation (1.1) to the isotropic subgroup of the origin in $G / P$ and this allows to transfer the $P$-invariant data of first and second order from the origin of $Q$ to the individual tangent spaces in all points of $M$.

We recall the details on the resulting groups

$$
G=\left(\left(\mathrm{SU}(2,1) / \mathbb{Z}_{3}\right) \times\left(\mathrm{SU}(2,1) / \mathbb{Z}_{3}\right)\right) \rtimes \mathbb{Z}_{2}
$$

in the hyperbolic case, and

$$
G=\left(\mathrm{SL}(3, \mathbb{C}) / \mathbb{Z}_{3}\right) \rtimes \mathbb{Z}_{2}
$$

in the elliptic case, $P, G_{0}$, and their Lie algebras in Appendix B. At the moment, let us notice that in both cases the Lie algebra $\mathfrak{g}$ carries the $|2|$-grading $\mathfrak{g}=\mathfrak{g}_{-2} \oplus \mathfrak{g}_{-1} \oplus$ $\mathfrak{g}_{0} \oplus \mathfrak{g}_{1} \oplus \mathfrak{g}_{2}, \mathfrak{p}=\mathfrak{g}_{0} \oplus \mathfrak{g}_{1} \oplus \mathfrak{g}_{2}$. In the elliptic case, this grading is just given by the five diagonals in the matrices in $S L(3, \mathbb{C})$ with $\mathfrak{g}_{-2}$ corresponding the entry at the lower left corner of the matrices, etc. This grading survives in the real form $S U(2,1)$. See e.g. [8] or [4] for more details on the grading. The subgroups $P$ and $G_{0}$ corresponding to $\mathfrak{p}$ and $\mathfrak{g}_{0}$ have all properties discussed in 2.1 below. In particular $P$ is the subgroup of all elements whose adjoint action leaves the $\mathfrak{p}$-submodules in $\mathfrak{g}$ invariant, while $G_{0}$ consists of all elements which leave the components $\mathfrak{g}_{i}$ invariant. Thus, the tangent space $T_{x} M$ at each point $x \in M$ is identified with the $P$-module $\mathfrak{g} / \mathfrak{p}$ which is the tangent space to the osculating quadric $Q$ at its origin, the normalized osculation transfers the $P$ submodule $\mathfrak{g}_{-1} \subset \mathfrak{g} / \mathfrak{p}$ to $T^{C R} M \subset T M$, and the algebraic structure of $\mathfrak{g} / \mathfrak{p}$ is carried over to the associated graded tangent space $\operatorname{Gr} T M=\left(T M / T^{C R} M\right) \oplus T^{C R} M$.

LEMMA 1.1. Let $M \subset \mathbb{C}^{4}$ be a hyperbolic or elliptic 6-dimensional CR-manifold. Then all algebraic brackets $T^{C R} M \times T^{C R} M \rightarrow T^{C R} M$ and $T^{C R} M \times T^{C R} M \rightarrow$ $T M / T^{C R} M$ on the real graded tangent space $\operatorname{Gr} T M$, and the analogous algebraic brackets on the complexified graded tangent space $\operatorname{Gr} T_{\mathbb{C}} M$ are obtained via the osculation from the corresponding brackets at the origin of the quadric.

In particular, the algebraic Lie bracket $\{,\}_{\text {Lie }}$ on $\operatorname{Gr} T M$ coincides with the algebraic bracket carried over by the osculation (1.1).

Proof. The Lie bracket on $\mathfrak{g}_{-}=\mathfrak{g}_{-2} \oplus \mathfrak{g}_{-1}$ is $G_{0}$-equivariant, and so the osculation (1.1) induces an algebraic bracket on the associated graded vector bundle $\operatorname{Gr} T M$. A neighborhood of the origin in $Q$ can be identified with the exponential image of $\mathfrak{g}_{-}$ in $G$ and the Lie bracket in $\mathfrak{g}_{-}$is given by the usual Lie brackets of the left invariant vector fields on $G$. By means of the osculation, we can project these fields onto $M$ locally and clearly the algebraic bracket $T^{C R} M \times T^{C R} M \rightarrow T M / T^{C R} M$ induced by the Lie bracket of vector fields on $M$ coincides with that one carried over from $\mathfrak{g}_{-}$by the osculation. Obviously, the result will not be effected by the action of an element in $P_{+}$on $Q$ (i.e. by the possible change of the osculation).

All other algebraic brackets on the real graded tangent space can be treated in exactly the same way, provided they are $P$-invariant on the quadric. The action of an element of $G_{0}$ always commutes with the osculation while the action of $P_{+}$is not 
visible in all our cases. Indeed, the action is trivial if all arguments and values are in $T^{C R} M$, while the contributions of the action is factored out in the case of the brackets $T^{C R} M \times T^{C R} M \rightarrow T M / T^{C R} M$. Similarly, the left invariant vector fields in the complexified tangent spaces on the quadric can be mapped into complex vector fields on $M$ and the above arguments apply as well.

Let us notice, however, that the possible algebraic brackets taking some arguments in $T Q / T^{C R} Q$ are never $P$-invariant.

The latter lemma turns out to be the most crucial point for our further development. Indeed, there is the general theory of the so called parabolic geometries which we adapt for our purposes in the next section. In particular, Theorem 2.13 due to [24, 4] will provide the canonical principal bundles together with canonical Cartan connections for all hyperbolic and elliptic 6-dimensional CR-manifolds with CR-codimension two (see the beginning of Section 2 for definitions and more explanation). We should also like to mention already now that the complete proof of Theorem 2.13 is in fact constructive, it is based on well known facts from representation theory, and it is even shorter and simpler than the ad hoc construction of the absolute parallelisms in [9]. The ultimate results read as follows:

THEOREM 1.2. On each 6-dimensional hyperbolic $C R$-manifold $M \subset \mathbb{C}^{4}$ of $C R$ codimension two, there is the unique normal Cartan connection $\omega$ of type $(G / P)$ on the principal fibre bundle $\mathcal{G} \rightarrow M$, up to isomorphisms. The subgroup $P$ is the subgroup of all elements in $G$ from (1.5) which respect the $\mathfrak{p}$-module filtration on $\mathfrak{s u}(2,1) \oplus \mathfrak{s u}(2,1)$.

THEOREM 1.3. On each 6-dimensional elliptic CR-manifold $M \subset \mathbb{C}^{4}$ of $C R$ codimension two, there is the unique normal Cartan connection $\omega$ of type $(G / P)$ on the principal fibre bundle $\mathcal{G} \rightarrow M$, up to isomorphisms. The subgroup $P$ is the subgroup of all elements in $G$ from (1.6) which respect the $\mathfrak{p}$-module filtration on $\mathfrak{s l}(3, \mathbb{C})$.

For the proof of these theorems see 2.14 below. The reason why the methods of [9] could not produce a principal fibre bundle $\mathcal{G}$ with structure group $P$ and a normal Cartan connection on $\mathcal{G}$, was hidden in the initial choice of the normalization which had to produce a Cartan connection without torsion. In our approach, the torsions are the important parts of the curvature which are easily observable on the CR-manifold itself. The Sections 3 and 4 are basically dealing with the consequences of the vanishing of the individual components of the torsion of the canonical Cartan connection for the hyperbolic and elliptic local geometries.

2. Parabolic geometries modeled over $|2|$-graded Lie algebras. The aim of this section is to introduce the reader to the so called parabolic geometries, but we shall concentrate on the cases similar to the real forms of the two-graded complex Lie algebra $\mathfrak{g}=\mathfrak{s l}(3, \mathbb{C}) \oplus \mathfrak{s l}(3, \mathbb{C})$. Beside well known facts, we shall also have to adapt and extend some points.

Let us notice first that the general ideas go back to E. Cartan and his notion of "espace generalisé". The interest in the parabolic structure groups was pointed out by Fefferman, [12], in connection with problems in conformal and CR geometries. Extensive study was undertaken even earlier by Tanaka (see [24] and the references therein), motivated by a class of equivalence problems for differential systems. Tanaka's approach was developed further, see e.g. [18, 27]. Motivation coming from twistor calculus led to another direction of related research, see e.g. [2, 1, 14]. General background and an introduction to the subject may be also found in [20]. The exposition in this section extends the development in [5,4] and follows mainly [21]. 
2.1. Graded Lie algebras. Let $\mathfrak{g}$ be a $|2|$-graded Lie algebra, $\mathfrak{p}$ and $\mathfrak{g}_{-}$its subalgebras:

$$
\begin{gathered}
\mathfrak{g}=\mathfrak{g}_{-2} \oplus \mathfrak{g}_{-1} \oplus \mathfrak{g}_{0} \oplus \mathfrak{g}_{1} \oplus \mathfrak{g}_{2} \\
\mathfrak{g}_{-}=\mathfrak{g}_{-2} \oplus \mathfrak{g}_{-1}, \quad \mathfrak{p}=\mathfrak{g}_{0} \oplus \mathfrak{g}_{1} \oplus \mathfrak{g}_{2}
\end{gathered}
$$

Further, let $G$ be a Lie group with the Lie algebra $\mathfrak{g}$. Then there is the subgroup $P \subset G$ of elements whose adjoint representations on $\mathfrak{g}$ preserve the filtration by $\mathfrak{p}$ submodules $\mathfrak{g}_{i} \oplus \mathfrak{g}_{i+1} \oplus \cdots \oplus \mathfrak{g}_{2}$ and there also is the subgroup $G_{0} \subset P$ of all elements whose adjoint representation leaves invariant all $\mathfrak{g}_{i}$. Thus the components $\mathfrak{g}_{i}$ of the grading can be understood as $G_{0}$-submodules, but also as the factors in the graded $P$-module components associated to the $P$-module filtration. Similarly we define the $|k|$-graded algebras $\mathfrak{g}=\mathfrak{g}_{-k} \oplus \cdots \oplus \mathfrak{g}_{k}$.

In the sequel, we shall deal with semi-simple $|2|$-graded Lie algebras exclusively. It is well known that all graded semi-simple Lie algebras are sums of $|k|$-graded algebras for suitable $k$ 's and the subgroups $P \subset G$ are always suitable real forms of parabolic subgroups $P_{\mathbb{C}} \subset G_{\mathbb{C}}$ in the complexification. The exposition below extends easily to general semi-simple $|k|$-graded Lie algebras and the corresponding parabolic structures, as discussed in [4] for example. Many geometric and algebraic properties of these geometries are deduced in [6].

2.2. Cartan connections. Let $G$ be any Lie group and $P$ be its closed subgroup. The left Maurer-Cartan form $\omega \in \Omega^{1}(G, \mathfrak{g})$ on the homogeneous space $p: G \rightarrow$ $G / P$ is the (homogeneous) prototype of the so called Cartan geometry. In general, a Cartan gsometry of type $G / P$ is a principal fibre bundle $p: \mathcal{G} \rightarrow M$ over a smooth manifold $M$, equipped with a $\mathfrak{g}$-valued one-form $\omega \in \Omega^{1}(\mathcal{G}, \mathfrak{g})$ satisfying

- $\omega\left(\zeta_{X}(u)\right)=X$ for all $X \in \mathfrak{p}$ and fundamental fields $\zeta_{X}$ on $\mathcal{G}$,

- $\omega$ is equivariant with respect to the right action $r^{b}$ on $\mathcal{G}$, i.e. $\left(r^{b}\right)^{*} \omega=$ $\operatorname{Ad}\left(b^{-1}\right) \circ \omega$ for all $b \in P$,

- the restrictions $\omega_{\mid T_{u} \mathcal{G}}: T_{u} \mathcal{G} \rightarrow \mathfrak{g}$ are linear isomorphisms, i.e. the obvious mapping $T \mathcal{G} \rightarrow \mathcal{G} \times \mathfrak{g}$ is a diffeomorphism.

See e.g. the recent book [20, pp. 181-184] for a detailed exposition.

The homomorphisms of Cartan geometries are those principal fibre bundle morphisms which respect the Cartan connections. The flat Cartan geometry is the homogeneous space $G \rightarrow G / P$ with the Maurer-Cartan form $\omega$.

Let us also observe that the above absolute parallelisms $\omega$ turn out to be special cases of principal connections $\tilde{\omega}$ on the principal bundle $\tilde{\mathcal{G}}=\mathcal{G} \times_{P} G$ with structure group $G$. Indeed, the connection forms of all principal connections on $\tilde{\mathcal{G}}$ whose horizontal distributions do not meet the tangent space $T \mathcal{G} \subset T \tilde{\mathcal{G}}$ restrict to forms $\omega$ with the required properties. See e.g. [20] for more comments.

2.3. Normal coordinates. For each $X \in \mathfrak{g}$, the parallelism $\omega$ defines the vector field $\omega^{-1}(X)$ on $\mathcal{G}$. The horizontal vector fields $\omega^{-1}(X)$ on $\mathcal{G}$ are those with $X \in \mathfrak{g}_{-}$ and their values span the horizontal distribution on $\mathcal{G}$. Due to the third property of $\omega$, the choice of a frame $u \in \mathcal{G}$ defines an injective smooth mapping of a neighborhood of zero in $\mathfrak{g}_{-}$

$$
X \mapsto \mathrm{Fl}_{1}^{\omega^{-1}(X)}(u), \quad X \in \mathfrak{g}_{-}
$$

defined by means of the flows of the vector fields $\omega^{-1}(X)$. The tangent space of its image at $u$ belongs to the horizontal distribution on $\mathcal{G}$ and its composition with the 
projection $p: \mathcal{G} \rightarrow M$ defines the locally defined mapping

$$
\tilde{u}: \mathfrak{g}_{-} \rightarrow M, \quad X \mapsto p\left(\mathrm{Fl}_{1}^{\omega^{-1}(X)}(u)\right)
$$

which is diffeomorphic on a neighborhood of the origin. We call $\tilde{u}$ the normal coordinates on $M$ given by the frame $u$. At the same time, $\tilde{u}$ induces the local trivialization $\sigma_{u}$,

$$
\sigma_{u}: M \rightarrow \mathcal{G}, \quad \tilde{u}(Y) \mapsto \mathrm{Fl}_{1}^{\omega^{-1}(Y)}(u) \in \mathcal{G} .
$$

Clearly, the normal coordinates around a fixed point $x \in M$ are parameterized by elements in $P$ and they generalize the usual normal coordinates of affine connections on manifolds. The general concept of the normal coordinates has been introduced and studied in [21].

2.4. Chains. The notion of normal coordinates suggests a straightforward generalization of the geodetical curves. For each choice of $X \in \mathfrak{g}_{-}$and $u \in \mathcal{G}$ we define the 1-chain $\alpha^{u, X}: \mathbb{R} \rightarrow M$ on a neighborhood of $0 \in \mathbb{R}$ by

$$
\alpha^{u, X}(t)=p\left(\mathrm{Fl}_{t}^{\omega^{-1}(X)}(u)\right) .
$$

Clearly the tangent direction to the 1-chain $\alpha^{u, X}$ at its origin is the vector $T p \cdot \omega^{-1}(X)(u)$ since the tangent bundle $T M$ is identified with the associated bundle $\mathcal{G} \times{ }_{P}(\mathfrak{g} / \mathfrak{p})$ via the adjoint representation, $\{u, X\} \mapsto T p \cdot \omega^{-1}(X)$. In particular we see immediately that many different 1-chains may share the same tangent direction.

The 1-chains have been studied under various names like Cartan's circles or generalized circles, see e.g. [20], and the chains introduced by Chern and Moser for CR-geometries of codimension one are exactly the 1-chains with $X \in \mathfrak{g}_{-2}$. Since $\operatorname{dim} \mathfrak{g}_{-2}=1$ for these geometries, the latter 1-chains coincide with the chains defined below.

All 1-chains corresponding to a fixed frame $u$ yield exactly the normal coordinates with origin at $p(u)$ and the transformation rules for these coordinates under the change of $u$ may be quite complicated, in general. On the other hand, the 1-chains corresponding to the parameters $\{u, X\}$ with $X \in \mathfrak{g}_{-2}$ have very specific properties. We define the chain $\beta^{u}: \mathfrak{g}_{-2} \rightarrow M$ by the formula

$$
\beta^{u}(X)=p\left(\mathrm{Fl}_{1}^{\omega^{-1}(X)}(u)\right) .
$$

Thus the chains are parameterized submanifolds in $M$ of dimension $\operatorname{dim} \mathfrak{g}_{-2}$.

REMARK 2.5. The importance of the chains grows whenever they are given uniquely by their tangent directions in the origin. Another important question is whether two different chains may intersect nontrivially in each small neighborhood of their common origin. The answer to these questions is usually very easy because of the following equivalent definition of 1-chains by means of their developments into the associated bundle $F M=\tilde{\mathcal{G}} \times_{G}(G / P)$.

The principal connection $\tilde{\omega}$ on $\tilde{\mathcal{G}}$ provides the induced (generalized) connection on the bundle $F M$ and there is the canonical embedding of $M$ into $F M, p(u) \mapsto\{u,[e]\}$. Thus each curve $\alpha(t) \in M$ can be mapped by the parallel transport of $\tilde{\omega}$ into a curve $\tilde{\alpha}$ in the fibre over $\alpha(0)$. This curve $\tilde{\alpha}$ is called the development of the curve $\alpha$. Clearly, the germs of curves through $\alpha(0)$ are in bijective correspondence with the germs of their developments. 
Now, our definition of the 1-chains can be easily rephrased as follows. The 1chains are exactly the curves $\alpha$ whose developments $\tilde{\alpha}$ are given by one-parametric subgroups in $G$, i.e. $\tilde{\alpha}^{u, X}=\{u,[\exp t X]\}$. See e.g. [21] for more details.

Since our chains $\beta^{u}$ are obtained via 1-chains, all structural questions mentioned above are obtained by the discussion of the chains in the homogeneous case.

2.6. Curvature and torsion. The structure equation

$$
d \omega=-\frac{1}{2}[\omega, \omega]+K
$$

defines the $\mathfrak{g}$-valued horizontal 2 -form $K \in \Omega^{2}(\mathcal{G}, \mathfrak{g})$. If we evaluate the structure equation on two horizontal vector fields we obtain the so called frame form of the curvature, the equivariant function $\kappa \in C^{\infty}\left(\mathcal{G}, \Lambda^{2} \mathfrak{g}_{-}^{*} \otimes \mathfrak{g}\right)^{P}$

$$
\begin{aligned}
\kappa(u)(X, Y) & =K\left(\omega^{-1}(X), \omega^{-1}(Y)\right)(u) \\
& =[X, Y]-\omega\left(\left[\omega^{-1}(X), \omega^{-1}(Y)\right](u)\right) .
\end{aligned}
$$

The Cartan geometry is locally isomorphic to the flat one if and only if its curvature vanishes.

If $\mathfrak{g}$ is semi-simple, then $P$ is a parabolic subgroup of the semi-simple group $G$ and we then refer to the above geometries as to parabolic geometries of type $G / P$.

The curvature $\kappa$ has values in the space of cochains of the Lie algebra cohomology $H^{*}\left(\mathfrak{g}_{-}, \mathfrak{g}\right)$. The grading on $\mathfrak{g}$ induces the grading on the space of cochains. The homogeneous cochains of degree $k$ are those which map $\mathfrak{g}_{i} \wedge \mathfrak{g}_{j}$ into $\mathfrak{g}_{i+j+k}$ and this grading is respected by the Lie algebra cohomology differential $\partial$. For each cochain $\eta \in \Lambda^{k} \mathfrak{g}_{-} \otimes A$ with values in a $\mathfrak{g}_{-}$-module $A$ the differential is given by

$$
\begin{aligned}
\partial \eta\left(X_{0}, \ldots, X_{k}\right)= & \sum_{i=0}^{k}(-1)^{i} X_{i} . \eta\left(X_{0}, \hat{\ldots}, X_{k}\right) \\
& +\sum_{i<j}(-1)^{i+j} \eta\left(\left[X_{i}, X_{j}\right], X_{0}, \ldots, X_{k}\right)
\end{aligned}
$$

where the dot in the first summand means the $\mathfrak{g}_{-}-$module action while the hats denote the obvious omitions.

In particular, the whole curvature splits into the homogeneous parts $\kappa^{(k)}$

$$
\kappa=\sum_{k=-\ell+2}^{3 \ell} \kappa^{(k)}
$$

where $\ell=2$ is the length of the grading. On the other hand, we may split $\kappa$ according to its values. In particular, there is the torsion part $\kappa_{-}$with values in $\mathfrak{g}_{-}$

$$
\kappa=\sum_{i=-\ell}^{\ell} \kappa_{i} \quad \kappa_{-}=\kappa_{-\ell} \oplus \cdots \oplus \kappa_{-1} \quad \kappa_{\mathfrak{p}}=\kappa_{0} \oplus \cdots \oplus \kappa_{\ell} .
$$

The torsion has a simple geometrical meaning: Let us define the horizontal bracket $[\xi, \eta]_{h}$ on the space $\mathcal{X}_{h}(\mathcal{G})$ of all fields belonging to the horizontal distribution on $\mathcal{G}$ by the standard Lie bracket followed by horizontal projection. By the very definition, the torsion of $\omega$ vanishes if and only if the mapping $\mathfrak{g}_{-} \ni X \mapsto \omega^{-1}(X) \in \mathcal{X}_{h}(\mathcal{G})$ is a Lie algebra homomorphism. 
2.7. Regular and normal connections. We say that the parabolic geometry $(\mathcal{G}, \omega)$ is regular if $\kappa^{(k)}=0$ for all $k \leq 0$.

In the sequel, we shall always assume $\mathfrak{g}$ is semi-simple. Then there is the adjoint of the Lie algebra cohomology differential $\partial$, the codifferential $\partial^{*}: \Lambda^{k} \mathfrak{g}_{-}^{*} \otimes \mathfrak{g} \rightarrow$ $\Lambda^{k-1} \mathfrak{g}_{-}^{*} \otimes \mathfrak{g}$.

We say that $\omega$ is a normal Cartan connection if its curvature is co-closed, i.e.

$$
\partial^{*} \circ \kappa=0 \in C^{\infty}\left(\mathcal{G}, \mathfrak{g}_{-}^{*} \otimes \mathfrak{g}\right) .
$$

Let us recall, that the whole space of cochains decomposes into a sum of irreducible components as a $\mathfrak{g}_{0}$-module. Each such component is either in the image of $\partial$ or in the image of $\partial^{*}$ or in the kernel of both. The latter components are called harmonic and they are in bijective correspondence with the non-zero cohomologies $H^{*}\left(\mathfrak{g}_{-}, \mathfrak{g}\right)$.

THEOREM 2.8. ([24, 27, 4]) Let $(\mathcal{G}, \omega)$ be a normal Cartan connection and assume that all components $\kappa^{(j)}, j<k$, vanish. Then $\partial \circ \kappa^{(k)}$ vanishes and so all non-trivial irreducible components of $\kappa^{(k)}$ are harmonic.

In particular, the whole curvature of $\omega$ vanishes if and only if its harmonic part does.

The latter theorem is a straightforward consequence of the important Bianchi identity for Cartan geometries:

$$
\begin{aligned}
\partial \kappa^{(k)}(X, Y, Z)= & -\sum_{\text {cyclic }} \sum_{i=1}^{k-1} \kappa^{(k-i)}\left(\kappa^{(i)}(X, Y), Z\right) \\
& \left.-\sum_{\text {cyclic }} \mathcal{L}_{\omega^{-1}(Z)} \kappa^{(k+|Z|)}(X, Y)\right)
\end{aligned}
$$

where the sum is the cyclic sum over $X, Y, Z \in \mathfrak{g}_{-}$, and $|Z|=j$ if $Z \in \mathfrak{g}_{j}$. See e.g. [4] for more details.

2.9. The underlying geometry on $M$. A part of the Cartan geometry $(\mathcal{G}, \omega)$ is visible directly on the underlying manifold $M$ and, fortunately, these data are sufficient in order to reconstruct the Cartan connection completely. This is the core of our approach to the CR structures in this paper. As before we shall restrict ourselves to the $|2|$-graded cases below, but the discussion extends easily to the general case.

The $P$-module structure on $\mathfrak{g}$ (defined via the Ad representation) determines the filtration by $P$-submodules

$$
\begin{gathered}
\mathfrak{g}=V^{-2} \supset V^{-1} \supset V^{0} \supset V^{1} \supset V^{2}=\mathfrak{g}_{2} \\
V^{k}=\mathfrak{g}_{k} \oplus \cdots \oplus \mathfrak{g}_{2} \subset \mathfrak{g}, \quad k=-2,-1,0,1,2 .
\end{gathered}
$$

This in turn defines the filtration on $T \mathcal{G}$

$$
\begin{gathered}
T \mathcal{G}=T^{-2} \mathcal{G} \supset T^{-1} \mathcal{G} \supset T^{0} \mathcal{G} \supset T^{1} \mathcal{G} \supset T^{2} \mathcal{G} \\
T_{u}^{k}(\mathcal{G})=\omega^{-1}(u)\left(V^{k}\right), \quad k=-2,-1,0,1,2, u \in \mathcal{G}
\end{gathered}
$$

The right invariance of $\omega$ yields

$$
\omega^{-1}(u \cdot b)(X)=T r^{b} \cdot \omega^{-1}(u)(\operatorname{Ad}(b) . X)
$$


where $T r^{b}$ denotes the tangent mapping to the right principal action of $b \in P$ on $\mathcal{G}$, and so the latter filtration on $T \mathcal{G}$ is $P$-invariant. The $P$-invariant projection $p: \mathcal{G} \rightarrow M$ defines then the filtration

$$
T M=T^{-2} M \supset T^{-1} M \supset\{0\}
$$

Moreover, each fixed frame $u \in \mathcal{G}$ with $p(u)=x \in M$ determines the linear isomorphism of filtered vector spaces

$$
\hat{u}: \mathfrak{g}_{-} \rightarrow T_{x} M \quad X \mapsto T p \cdot \omega^{-1}(X)(u)
$$

and on the level of the associated graded spaces we obtain the linear isomorphism

$$
\hat{u}: V^{-2} / V^{-1} \oplus V^{-1} / V^{0} \simeq \mathfrak{g}_{-2} \oplus \mathfrak{g}_{-1} \rightarrow T_{x}^{-2} M / T_{x}^{-1} M \oplus T_{x}^{-1} M .
$$

The whole structure group $P$ is a semidirect product of its reductive subgroup $G_{0}$ (corresponding to the Lie algebra $\mathfrak{g}_{0}$ ) and the subgroup $P_{+}$which corresponds to $\mathfrak{p}_{+}=\mathfrak{g}_{1} \oplus \mathfrak{g}_{2}$. Obviously, the latter identification $\hat{u}$ does not change if we replace the frame $u$ by $u . b$ with $b \in P_{+}$. Thus we have identified the graded tangent bundle $\operatorname{Gr} T M$ with the associated vector bundle to the principal bundle $\mathcal{G}_{0}=\mathcal{G} / P_{+}$whose standard fibre is the $G_{0}$-module $\mathfrak{g}_{-}$. In particular the Lie bracket on $\mathfrak{g}_{-}$is transfered to the algebraic bracket $\{,\}_{0}$ by

$$
\left\{\xi_{x}, \eta_{x}\right\}_{0}=\hat{u}\left(\left[\hat{u}^{-1}\left(\xi_{x}\right), \hat{u}^{-1}\left(\eta_{x}\right)\right]\right), \quad \xi_{x}, \eta_{x} \in \operatorname{Gr} T_{x} M, u \in \mathcal{G} .
$$

Notice that this definition does not depend on the choice of $u$ since $\hat{u}$ is independent of the action of $P_{+}$and the Lie bracket on $\mathfrak{g}_{-}$is $G_{0}$-equivariant. Since our $G_{0}$-structure on $\operatorname{Gr} T M$ is defined by the Cartan connection, we may choose representing vectors $\bar{\xi}_{x} \in T_{x}^{i} M, \bar{\eta}_{x} \in T_{x}^{j} M$, their covering vectors $\hat{\xi}_{u}, \hat{\eta}_{u} \in T \mathcal{G}$ and we obtain

$$
\left\{\xi_{x}, \eta_{x}\right\}_{0}=\pi\left(\omega^{-1}\left(\left[\omega\left(\hat{\xi}_{u}\right), \omega\left(\hat{\eta}_{u}\right)\right]\right)(u)\right)
$$

where $\pi$ is the obvious projection $T^{i+j} \mathcal{G} \rightarrow T^{i+j} M \rightarrow T^{i+j} M / T^{i+j+1} M$.

We shall see in a while that the regular Cartan geometries are exactly those for which the latter bracket is induced from the Lie bracket of vector fields in an algebraic way. Since we shall need a good control over the relations between the brackets of the horizontal vector fields and some brackets on the underlying manifold in its proof, we shall first formulate a general lemma based on our concept of the normal coordinates.

Lemma 2.10. Let $u \in \mathcal{G}$ and let $\sigma_{u}$ be the corresponding distinguished local trivialization of $\mathcal{G}$, see (2.3). Further let $X, Y \in \mathfrak{g}_{-}$, and consider the projectable vector fields $\tilde{\xi}, \tilde{\eta}$ over $M$, such that their restrictions to the image of $\sigma_{u}$ coincide with the horizontal fields $\omega^{-1}(X), \omega^{-1}(Y)$, respectively. Then $\left[\omega^{-1}(X), \omega^{-1}(Y)\right](u)=[\tilde{\xi}, \tilde{\eta}](u)$.

Thus, in particular

$$
T p \cdot\left[\omega^{-1}(X), \omega^{-1}(Y)\right](u)=[T p . \tilde{\xi}, T p \cdot \tilde{\eta}](p(u))
$$

Proof. Let us write $\tilde{\xi}=\omega^{-1}(X)+\mu, \tilde{\eta}=\omega^{-1}(Y)+\nu$ and compute their bracket. 
By the very definition, we obtain

$$
\begin{aligned}
{[\tilde{\xi}, \tilde{\eta}](u) } & =\left.\frac{d}{d t}\right|_{0} T\left(\mathrm{Fl}_{-t}^{\tilde{\xi}}\right) \circ\left(\omega^{-1}(Y)+\nu\right) \circ\left(\mathrm{Fl}_{t}^{\tilde{\xi}}\right)(u) \\
& =\left.\frac{d}{d t}\right|_{0} T\left(\mathrm{Fl}_{-t}^{\tilde{\xi}}\right) \circ\left(\omega^{-1}(Y)+\nu\right) \circ\left(\mathrm{Fl}_{t}^{\omega^{-1}(X)}\right)(u) \\
& =\frac{d}{d t} T\left(\mathrm{Fl}_{-t}^{\tilde{\xi}}\right) \circ\left(\omega^{-1}(Y)\right) \circ\left(\mathrm{Fl}_{t}^{\omega^{-1}(X)}\right)(u) \\
& =\left[\tilde{\xi}, \omega^{-1}(Y)\right](u)
\end{aligned}
$$

where the first equality follows from the fact that the flows of $\tilde{\xi}$ and $\omega^{-1}(X)$ through $u$ coincide, the next one results from the vanishing of $\nu$ on the image of $\sigma_{u}$. Now, repeating the same arguments for $\left[\omega^{-1}(Y), \tilde{\xi}\right]$, we achieve just the required equality.

LEMMA 2.11. Let $\omega \in \Omega^{1}(\mathcal{G}, \mathfrak{g})$ be a Cartan connection with a $|2|$-graded Lie algebra $\mathfrak{g}$. Then $\kappa^{(i)}=0$ for all $i<0$ and the Lie bracket of vector fields defines an algebraic bracket $\{,\}_{\text {Lie }}$ on the graded vector bundle $\operatorname{Gr} T M$. Moreover, $\kappa^{(0)}$ vanishes if and only if the latter bracket coincides with the algebraic bracket $\{,\}_{0}$ on $\operatorname{Gr} T M$.

Proof. Recall that the defining equation for the homogeneous components $\kappa^{(k)}(u)(X, Y), k \neq 0, u \in \mathcal{G}, X \in \mathfrak{g}_{i}, Y \in \mathfrak{g}_{j}$ is

$$
\kappa^{(k)}(u)(X, Y)=-\omega_{i+j+k}\left(\left[\omega^{-1}(X), \omega^{-1}(Y)\right](u)\right)
$$

while the component of degree zero is

$$
\kappa^{(0)}(u)(X, Y)=[X, Y]-\omega_{i+j}\left(\left[\omega^{-1}(X), \omega^{-1}(Y)\right](u)\right)
$$

Now, consider vector fields $\xi$ in $T^{i} M, \eta$ in $T^{j} M$ and let us choose elements $X_{r} \in V^{i}$, $Y_{s} \in V^{j}$ such that $\xi=T p \cdot \sum_{r} f^{r} \omega^{-1}\left(X_{r}\right), \eta=T p \cdot \sum_{s} g^{s} \omega^{-1}\left(Y_{s}\right)$ with suitable functions $f^{r}, g^{s}$ on $\mathcal{G}$. Then

$$
[\xi, \eta]=\left(T p \cdot \sum_{r, s} f^{r} g^{s}\left[\omega^{-1}\left(X_{r}\right), \omega^{-1}\left(Y_{s}\right)\right]\right) \bmod T^{i+j+1} M
$$

The negative homogeneous components $\kappa^{(k)}, k<0$ have to vanish because the algebra is $|2|$-graded and so we have no choice of arguments for cochains with such homogeneity. The lowest possible case will be a cochain $\mathfrak{g}_{-1} \times \mathfrak{g}_{-1} \rightarrow \mathfrak{g}_{-2}$ of homogeneity zero. The fact that the Lie bracket of vector fields produces an algebraic bracket on the associated graded tangent bundle is obvious.

Now, the two brackets in question may be expressed for all vectors $\xi_{x}=\pi\left(\omega^{-1}(X)(u)\right)$ and $\eta_{x}=\pi\left(\omega^{-1}(Y)(u)\right)$ as

$$
\begin{aligned}
\left\{\xi_{x}, \eta_{x}\right\}_{0} & =\pi\left(\omega^{-1}([X, Y])(u)\right) \\
\left\{\xi_{x}, \eta_{x}\right\}_{\text {Lie }} & =[T p . \tilde{\xi}, T p . \tilde{\eta}](x) \bmod T^{i+j+1} M \\
& =\pi\left(\left[\omega^{-1}(X), \omega^{-1}(Y)\right](u) \bmod T^{i+j+1} \mathcal{G}\right) \\
& =\pi\left(\omega^{-1}\left(\omega_{i+j}\left(\left[\omega^{-1}(X), \omega^{-1}(Y)\right](u)\right)\right)\right)
\end{aligned}
$$

where $\tilde{\xi}$ or $\tilde{\eta}$ are some projectable fields from the previous Lemma 2.10. Thus, according to (2.8), the two brackets equal each other if and only if $\kappa^{(0)}$ vanishes. 
Now we have got the motivation for the following definition of geometric structures on manifolds. Let us also remark that the version of the latter lemma which is valid for all $|k|$-graded structures needs one more condition. Namely, the existence of the algebraic bracket induced by the Lie brackets of vector fields, which is equivalent to the vanishing of all negative components $\kappa^{(k)}, k<0$.

Definition 2.12. Let $\mathfrak{g}, G, P$, and $G_{0}$ be as in 2.1. A regular $(\mathfrak{g}, P)$-structure on a smooth manifold $M$ is a filtration of the tangent bundle $T M$

$$
T M=T^{-2} M \supset T^{-1} M
$$

together with the reduction of the structure group of the associated graded tangent vector bundle $\operatorname{Gr} T M$ to the subgroup $G_{0}$, such that the algebraic bracket on $\operatorname{Gr} T M$ induced by the Lie bracket of vector fields coincides with the algebraic Lie bracket defined by the $G_{0}$-structure.

We may understand the above condition as the requirement that the subbundle $T^{-1} M$ be reasonably non-involutive. Due to our restriction to $|2|$-graded algebras we do not need to consider the other condition from Lemma 2.11 on the Lie brackets of vector fields, namely that they must not be "too much non-involutive".

Surprisingly enough there is the theorem claiming that, apart from a few exceptions, all regular normal parabolic geometries are uniquely given by the underlying $(\mathfrak{g}, P)$-structures on the manifolds $M$ :

THEOREM 2.13. Let $M$ be a smooth manifold, $\mathfrak{g}$ a graded semi-simple Lie algebra, $G$ a Lie group with Lie algebra $\mathfrak{g}$, and assume that all homogeneous components of the cohomologies $H_{\ell}^{1}\left(\mathfrak{g}_{-}, \mathfrak{g}\right)$ with positive degrees $\ell>0$ are trivial. Then there is a bijective equivalence between isomorphism classes of the regular $(\mathfrak{g}, P)$-structures on $M$ and the isomorphism classes of regular normal Cartan geometries $(\mathcal{G}, \omega)$ over $M$.

For the proof see Section 3 of [4]. The computations in [27, 4] show that, apart from situations with simple components in $\mathfrak{g}_{0}$, the only exceptions are $\mathfrak{g}=\mathfrak{s l}(2, \mathbb{C})$, specific maximal parabolic subalgebras in special linear algebras in higher dimension (|1|-graded examples) and specific maximal subalgebras in symplectic algebras $(|2|-$ graded examples). An equivalent theorem for the cases $\mathfrak{g}$ simple and $G$ connected was proved in [24].

2.14. Proof of Theorems 1.2 and 1.3. The relevant cohomologies for the real forms of $\mathfrak{s l}(3, \mathbb{C}) \oplus \mathfrak{s l}(3, \mathbb{C})$ are computed in Appendix A. In particular, there is no obstruction in the construction of the normal Cartan connections out of regular $(\mathfrak{g}, P)$ structures according to Theorem 2.13. The definition of the relevant $(\mathfrak{g}, P)$-structures by means of the fundamental second order osculation (1.1) was discussed at the end of Section 1, see Lemma 1.1.

3. The hyperbolic structures. In this section, we shall study the consequences of the algebraic structure of $\mathfrak{s u}(2,1) \oplus \mathfrak{s u}(2,1)$ for the hyperbolic points on embedded 6-dimensional CR-manifolds of CR-codimension two $M \subset \mathbb{C}^{4}$. Thus the Lie groups $G$, $P, G_{0}$, as well as the corresponding Lie algebras will be fixed throughout this section.

3.1. Almost product and almost complex structures. As we noticed already in the proof of Theorem 1.2 , there is the relevant $(\mathfrak{g}, P)$-structure on $M$. Since the individual left and right components of $\mathfrak{g}$ are $P$-submodules up to swapping $\mathrm{L}$ and $\mathrm{R}$, this structure introduces the natural splitting of the whole tangent bundle $T M$, i.e. an almost product structure on $M$. The almost product structure also restricts to 
the complex tangent bundles $T^{C R} M$. Following our notation for the left and right components of the structure group in the Appendix, we shall write

$$
\begin{gathered}
T M=T^{R} M \oplus T^{L} M, \quad T^{\mathrm{CR}} M=T^{\mathrm{CR}, R} M \oplus T^{\mathrm{CR}, L} M \\
\operatorname{Gr} T M=\left(T^{L} M / T^{\mathrm{CR}, L} M \oplus T^{\mathrm{CR}, L} M\right) \oplus\left(T^{R} M / T^{\mathrm{CR}, R} M \oplus T^{\mathrm{CR}, R} M\right)
\end{gathered}
$$

but we keep in mind that the splitting is available only locally, in general. In particular, the two components of $T M$ are orthogonal with respect to the algebraic bracket $\{,\}_{\text {Lie }}$.

Next, we observe that the canonical almost complex structure $J$ defined on $T^{C R} M$ is induced by the $(\mathfrak{g}, P)$-structure. Indeed, we define

$$
J \in\left(T^{C R} M\right)^{*} \otimes T^{C R} M, \quad J\left(T p \cdot \omega^{-1}(X)(u)\right)=T p \cdot\left(\omega^{-1}(i X)(u)\right)
$$

and this formula does not depend on the choice of $u$ and $X$ because the adjoint action of $P$ on $\mathfrak{g}_{-1} \subset \mathfrak{g} / \mathfrak{p}$ is complex linear.

At the same time, there is the obvious integrable complex structure coming from the definition $T^{C R} M=T M \cap i T M \subset T \mathbb{C}^{4}$ on the embedded CR-manifolds. The fundamental osculation (1.1) then implies that these two almost complex structures on $T^{C R} M$ coincide.

3.2. The abstract hyperbolic CR-manifolds of dimension six and CR-codimension two are defined by the specification of a regular $(\mathfrak{g}, P)$-structure on $M$ in the sense of Definition 2.12. In particular, they come equipped by the CR-subbundle $T^{\mathrm{CR}} M \subset T M$ of real codimension two with an almost complex structure, and the compatible almost product structure on $T M$. The general theory then applies as well and so the normal Cartan connections are given uniquely on all such manifolds. We shall see, however, that the embedded ones have very specific features. The automatic integrability of the almost complex structure $J$ on $T^{C R} M$ is an example. We can meet these more general structures on some 6-dimensional real submanifolds in 8-dimensional almost complex manifolds.

Our goal is to understand fully the local geometrical properties. For that reason we shall first discuss all possible algebraic brackets on $T M$ which arise from the Lie bracket of vector fields and we shall link them to certain components of the curvature of the canonical Cartan connection $\omega$ on $M$. In fact we shall work on the abstract level, forgetting more or less about the embedding of the manifold $M$ into $\mathbb{C}^{4}$. For the embedded hyperbolic CR-manifolds, however, some of the obstructions will vanish automatically.

For example, the algebraic Lie bracket of two vector fields $\xi, \eta$ in $T^{\mathrm{CR}, L} M$ has no contribution in $T^{R} M / T^{C R, R} M$ and so the projection of the Lie bracket $[\xi, \eta]$ to $T^{R} M=T M / T^{L} M$ has values in $T^{\mathrm{CR}, R} M$. Analogously we can deal with left and right components exchanged and so there are two obvious algebraic brackets

$$
\begin{aligned}
& \{,\}_{L}: T^{\mathrm{CR}, L} M \times T^{\mathrm{CR}, L} M \rightarrow T^{\mathrm{CR}, R} M \\
& \{,\}_{R}: T^{\mathrm{CR}, R} M \times T^{\mathrm{CR}, R} M \rightarrow T^{\mathrm{CR}, L} M
\end{aligned}
$$

which have to vanish automatically for all embedded hyperbolic CR-manifolds in view of Lemma 1.1. We shall see in a moment that these brackets vanish even for the abstract structures.

Our general strategy will be to link algebraic brackets to certain components of the curvature $\kappa$ of the Cartan connection $\omega$. According to Theorem 2.8, we have to 


\begin{tabular}{|c|c|l|}
\hline homog. & cochains & comment \\
\hline 1 & $\mathfrak{g}_{-2}^{R} \times \mathfrak{g}_{-1}^{R} \rightarrow \mathfrak{g}_{-2}^{L}$ & real linear in both arguments \\
1 & $\mathfrak{g}_{-2}^{L} \times \mathfrak{g}_{-1}^{L} \rightarrow \mathfrak{g}_{-2}^{R}$ & real linear in both arguments \\
\hline 1 & $\mathfrak{g}_{-1}^{L} \times \mathfrak{g}_{-1}^{R} \rightarrow \mathfrak{g}_{-1}^{L}$ & antilinear in both arguments \\
1 & $\mathfrak{g}_{-1}^{L} \times \mathfrak{g}_{-1}^{R} \rightarrow \mathfrak{g}_{-1}^{R}$ & sesquilinear \\
1 & $\mathfrak{g}_{-1}^{R} \times \mathfrak{g}_{-1}^{L} \rightarrow \mathfrak{g}_{-1}^{R}$ & antilinear in both arguments \\
1 & $\mathfrak{g}_{-1}^{R} \times \mathfrak{g}_{-1}^{L} \rightarrow \mathfrak{g}_{-1}^{L}$ & sesquilinear \\
\hline 4 & $\mathfrak{g}_{-2}^{L} \times \mathfrak{g}_{-1}^{L} \rightarrow \mathfrak{g}_{1}^{L}$ & real and complex linear \\
4 & $\mathfrak{g}_{-2}^{R} \times \mathfrak{g}_{-1}^{R} \rightarrow \mathfrak{g}_{1}^{R}$ & real and complex linear \\
\hline
\end{tabular}

TABLE 3.1. Real cohomologies of $\mathfrak{g}_{-}$with coefficients in $\mathfrak{g}$

start by the description of the real cohomologies

$$
H_{*}^{2}\left(\mathfrak{g}_{-}^{L} \oplus \mathfrak{g}_{-}^{R}, \mathfrak{s u}(2,1)^{L} \oplus \mathfrak{s u}(2,1)^{R}\right) .
$$

LEMMA 3.3. All irreducible components of these real cohomologies are the onedimensional $\mathfrak{g}_{0}$-modules which are generated by the (real) bilinear cochains listed in Table 3.1.

Proof. Let us consider the $\mathfrak{g}_{0}$-modules

$$
A_{\ell}=H_{\ell}^{2}\left(\mathfrak{g}_{-}^{L} \oplus \mathfrak{g}_{-}^{R}, \mathfrak{s u}(2,1)^{L} \oplus \mathfrak{s u}(2,1)^{R}\right) .
$$

By the general theory we know that the complexifications $\left(A_{\ell}^{*}\right)_{\mathbb{C}}$ of the dual $\mathfrak{g}_{0^{-}}$ modules $A_{\ell}^{*}$ are the complex cohomologies $H_{-\ell}^{2}\left(\mathfrak{p}_{+}, \mathfrak{s l}(3, \mathbb{C}) \oplus \mathfrak{s l}(3, \mathbb{C})\right)$ listed in the table of all complex cohomologies, see Table A.2 in Appendix A. Further, let us notice that the two components in $\mathfrak{g}_{-1}$ have a canonical complex structure. Now, we have just to keep in mind, that a complexification of a real linear mapping $\phi: V \rightarrow W$, defined on a complex vector space $V$, splits into two components according to the splitting of the complexification $V_{\mathbb{C}}=V \oplus \bar{V}$. If the target of such a mapping is complex as well, then the mapping $\phi$ itself splits into the complex linear and complex antilinear parts. Thus the complex cohomologies on the list of Table A.2, and the other half of them, must come exactly from the components listed in Table 3.1.

Now we are ready to find the geometric meaning of the individual torsion components. First, we shall focus on the obstructions against the integrability of the natural almost product structure on $M$.

Thus we are interested in brackets $\operatorname{Gr} T^{L} M \times \operatorname{Gr} T^{L} M \rightarrow \operatorname{Gr} T^{R} M$ and those with the left and right components exchanged. The restriction of $\{,\}_{\text {Lie }}$ vanishes clearly. Hence, apart from the algebraic brackets (3.1), (3.2), there is another candidate

$$
\begin{aligned}
& \{,\}_{L}: T^{L} M / T^{\mathrm{CR}, L} M \times T^{\mathrm{CR}, L} M \rightarrow T^{R} M / T^{\mathrm{CR}, R} M \\
& \{,\}_{R}: T^{R} M / T^{\mathrm{CR}, R} M \times T^{\mathrm{CR}, R} M \rightarrow T^{L} M / T^{\mathrm{CR}, L} M .
\end{aligned}
$$


Indeed, choosing any representative of the argument from the quotient space, the ordinary Lie bracket projected to the desired component yields our algebraic bracket. In contrast to the Levi form, these two algebraic brackets are not coming from the quadric by the osculation.

Lemma 3.4. The brackets (3.1), (3.2) vanish identically. The brackets (3.3), (3.4) are given by the formulae

$$
\begin{aligned}
& \left\{\pi_{L}(\xi), \eta\right\}_{L}=-\pi_{R}\left(T p \cdot \omega^{-1}\left(\kappa^{(1)}(u)(X, Y)\right)(u)\right) \\
& \left\{\pi_{R}(\xi), \eta\right\}_{R}=-\pi_{L}\left(T p \cdot \omega^{-1}\left(\kappa^{(1)}(u)(X, Y)\right)(u)\right)
\end{aligned}
$$

where $u \in \mathcal{G}, \pi_{L}$ and $\pi_{R}$ are the obvious quotient projections in the left and right components of the graded tangent space, and $X \in \mathfrak{g}_{-2}^{L}, Y \in \mathfrak{g}_{-1}^{L}$, or $X \in \mathfrak{g}_{-2}^{R}, Y \in \mathfrak{g}_{-1}^{R}$, respectively, and

$$
\xi=T p \cdot \omega^{-1}(X)(u), \quad \eta=T p \cdot \omega^{-1}(Y)(u) .
$$

There are no more non-trivial algebraic brackets $\operatorname{Gr} T^{L} M \times \operatorname{Gr} T^{L} M \rightarrow \operatorname{Gr} T^{R} M$ and $\operatorname{Gr} T^{R} M \times \operatorname{Gr} T^{R} M \rightarrow \operatorname{Gr} T^{L} M$.

Proof. We shall discuss only brackets $\operatorname{Gr} T^{L} M \times \operatorname{Gr} T^{L} M \rightarrow \operatorname{Gr} T^{R} M$. The other ones are treated analogously.

The first part is quite easy. Let us consider $\xi_{x}, \eta_{x} \in T_{x}^{\mathrm{CR}, L} M$. Further, choose $u \in \mathcal{G}, x=p(u)$, and $X, Y \in \mathfrak{g}_{-1}^{L}$ such that $\xi_{x}=T p \cdot \omega^{-1}(X)(u), \eta_{x}=T p \cdot \omega^{-1}(Y)(u)$. According to the Lemma 2.10, there are the projectable vector fields $\tilde{\xi}, \tilde{\eta}$ on $\mathcal{G}$ such that their projections $\xi=T p \circ \tilde{\xi}, \eta=T p . \tilde{\eta}$ satisfy $\xi(x)=\xi_{x}, \eta(x)=\eta_{x}$ and

$$
[\xi, \eta](x)=T p \cdot[\tilde{\xi}, \tilde{\eta}](u)=T p \cdot\left[\omega^{-1}(X), \omega^{-1}(Y)\right](u) .
$$

Now let us recall the general formulae (2.7) and (2.8) for the evaluations of curvatures and remember there are no curvature components of non-positive homogeneities. In particular,

$$
\omega\left(\left[\omega^{-1}(X), \omega^{-1}(Y)\right](u)\right) \in \mathfrak{g}_{-2}^{L} \oplus\left(\mathfrak{g}_{-1}^{L} \oplus \mathfrak{g}_{-1}^{R}\right) \bmod \mathfrak{p} .
$$

Thus applying the projection $\pi_{R}$ onto the image $T^{\mathrm{CR}, R} M$ of $\{,\}_{L}$, we may rewrite (3.7) as

$$
\begin{aligned}
\left\{\xi_{x}, \eta_{x}\right\}_{L} & =\pi_{R} \circ T p \cdot[\tilde{\xi}, \tilde{\eta}] \\
& =\pi_{R} \circ T p \cdot \omega^{-1}(u)\left(\omega\left(\left[\omega^{-1}(X), \omega^{-1}(Y)\right](u)\right)\right) \\
& =-\pi_{R} \circ T p \cdot \omega^{-1}(u)\left(\kappa^{(1)}(u)(X, Y)\right) .
\end{aligned}
$$

In particular, the bracket must vanish because there is no cohomology represented by cochains $\mathfrak{g}_{-1}^{L} \times \mathfrak{g}_{-1}^{L} \rightarrow \mathfrak{g}_{-1}^{R}$, see Table 3.1 , and so this component of the curvature vanishes by Theorem 2.8 .

We shall proceed analogously in the case of the bracket (3.3). Let us fix again a frame $u \in \mathcal{G}, x=p(u)$, choose the element in $T^{L} M / T^{\mathrm{CR}, L} M$ represented by $T p \cdot \omega^{-1}(X)(u)$ with $X \in \mathfrak{g}_{-2}^{L}$, and choose another vector $\eta_{x} \in T_{x}^{\mathrm{CR}, L} M, \eta_{x}=$ $T p \cdot \omega^{-1}(Y)(u)$, with $Y \in \mathfrak{g}_{-1}^{L}$. Next, we consider the projectable vector fields $\tilde{\xi}$ on $\mathcal{G}$ such that $\omega^{-1}(X)=\tilde{\xi}$ on the image of $\sigma_{u}$ and similarly for $\eta$. Then the value of $T p \circ \tilde{\xi}=\xi$ at $x$ represents the right argument in $T^{L} M / T^{C R, L} M$ and we obtain

$$
[\xi, \eta](x)=T p \cdot[\tilde{\xi}, \tilde{\eta}](u)=T p \cdot\left[\omega^{-1}(X), \omega^{-1}(Y)\right](u)
$$


(see again Lemma 2.10). Since $X \in \mathfrak{g}_{-2}^{L}, Y \in \mathfrak{g}_{-1}^{L}$, our table of cohomologies implies

$$
\kappa^{(1)}(u)(X, Y)=-\omega_{-2}^{R}\left(\left[\omega^{-1}(X), \omega^{-1}(Y)\right](u)\right) \in \mathfrak{g}_{-2}^{R}
$$

where $\omega_{-2}^{R}$ is the component of $\omega$ valued in $\mathfrak{g}_{-2}^{R}$. In particular we obtain the required equality (3.5).

There are still two more possibilities for algebraic brackets $\operatorname{Gr} T^{L} M \times \operatorname{Gr} T^{L} M \rightarrow$ $\operatorname{Gr} T^{R} M$. The first one,

$$
T^{L} M / T^{\mathrm{CR}, L} M \times T^{L} M / T^{\mathrm{CR}, L} M \rightarrow \operatorname{Gr} T^{R} M
$$

is obviously zero since the arguments are from an one-dimensional space. The remaining brackets

$$
\begin{aligned}
& \{,\}_{L}: T^{L} M / T^{\mathrm{CR}, L} M \times T^{\mathrm{CR}, L} M \rightarrow T^{\mathrm{CR}, R} M \\
& \{,\}_{R}: T^{L} M / T^{\mathrm{CR}, R} M \times T^{\mathrm{CR}, R} M \rightarrow T^{\mathrm{CR}, L} M
\end{aligned}
$$

can be well defined and are algebraic if and only if the brackets (3.3) and (3.4) vanish, respectively. If so, then their values are again defined by considering the representatives of the elements in the quotient spaces in the domain. By the vanishing assumption, their projection to the quotient on the right hand side is zero, thus they lie in the desired targets.

So let us assume that the bracket (3.3) vanishes. Then tracing the above computation of the latter bracket step by step, with the target replaced by $T^{\mathrm{CR}, R} M$, we end up with the formula

$$
\left\{\pi_{L}(\xi(x)), \eta(x)\right\}_{L}=-\pi_{R} \circ T p\left(\omega^{-1}(u)\left(\kappa^{(2)}(u)(X, Y)\right)\right) .
$$

Thus the vanishing of our bracket is equivalent to the vanishing of the corresponding component $\kappa^{(2)}: \mathfrak{g}_{-2}^{L} \times \mathfrak{g}_{-1}^{L} \rightarrow \mathfrak{g}_{-1}^{R}$. Consider now the homogeneous component of degree two of the Bianchi identity, see (2.5) in Section 2. Its right hand side includes terms of two kinds:

$$
\kappa^{(1)}\left(\kappa^{(1)}(X, Y), Z\right) \quad \mathcal{L}_{\omega^{-1}(Z)} \kappa^{(2+|Z|)}(X, Y)
$$

The differential $\partial \kappa^{(2)}$ on the left hand side is homogeneous of degree two again. Since our component of $\kappa^{(2)}$ is not in the list of the available cohomologies and $\kappa^{(2)}$ is coclosed, this component must be in the image of $\partial^{*}$. Further, let us notice that $\partial$ acts injectively on the image of $\partial^{*}$ (cf. the Hodge-structure mentioned in 2.7). Thus the image of $\kappa^{(2)}$ under $\partial$ vanishes if and only if this component vanishes too. Now, we are interested only in the component $\mathfrak{g}_{-2}^{L}{ }^{*} \otimes \mathfrak{g}_{-1}^{L}{ }^{*} \otimes \mathfrak{g}_{-1}^{R}$ and so its image under $\partial$ will sit in the subspace (cf. (2.4))

$$
\left(\mathfrak{g}_{-1}^{R *} \otimes \mathfrak{g}_{-2}^{L}{ }^{*} \otimes \mathfrak{g}_{-1}^{L}{ }^{*} \otimes \mathfrak{g}_{-2}^{R}\right) \oplus\left(\mathfrak{g}_{-1}^{L}{ }^{*} \otimes \mathfrak{g}_{-1}^{L}{ }^{*} \otimes \mathfrak{g}_{-1}^{L}{ }^{*} \otimes \mathfrak{g}_{-1}^{R}\right) .
$$

Our knowledge of all possibly non-zero components of first degree in $\kappa$ (remember we assume that the bracket (3.5) vanishes) and a straightforward inspection of the few possibilities of the placement of the arguments in the two terms in the Bianchi identity shows that there is no way to get anything non-zero.

Thus the vanishing of the last possible algebraic bracket has been proved.

THEOREM 3.5. Let $M$ be an abstract hyperbolic 6-dimensional CR-manifold of $C R$-codimension two. The left distribution $T^{L} M$ is involutive if and only if the bracket 
(3.3) vanishes, the right distribution is involutive if and only if the bracket (3.4) vanishes.

The almost product structure on $M$ is integrable if and only if both these brackets vanish.

Proof. All projections of the Lie brackets $T^{L} M \times T^{L} M \rightarrow T^{R} M$ are linear over functions and thus algebraic. Therefore, Lemma 3.4 implies immediately the first claim. Similarly for the other distribution $T^{R} M$ and the last claim follows by the standard foliation theory.

THEOREM 3.6. Let $M$ be a 6-dimensional abstract hyperbolic $C R$-manifold of $C R$ codimension two. The canonical almost complex structure $J$ on $T^{C R} M$ is integrable if and only if the part $\kappa_{a a}^{(1)} \in C^{\infty}\left(\mathcal{G}, \mathfrak{g}_{-1}^{*} \wedge \mathfrak{g}_{-1}^{*} \otimes \mathfrak{g}_{-1}\right)$ of $\kappa^{(1)}$ which is antilinear in both arguments vanishes. In particular, this part of the torsion vanishes on the embedded 6-dimensional hyperbolic CR-manifolds in $\mathbb{C}^{4}$.

Proof. By the defining properties of the regular $(\mathfrak{g}, P)$-structures, the complexified CR-tangent subbundle $T_{\mathbb{C}}^{C R} M \subset T_{\mathbb{C}} M$ must be involutive. Thus the obstruction against the integrability of $J$ is the Nijenhuis tensor $N \in \Lambda^{2}\left(T^{C R} M\right)^{*} \otimes T^{C R} M$. Consequently, the theorem will be proved once we verify the following claim: The Nijenhuis tensor $N$, expressed by its frame form $\nu \in C^{\infty}\left(\mathcal{G}, \mathfrak{g}_{-1}^{*} \wedge \mathfrak{g}_{-1}^{*} \otimes \mathfrak{g}_{-1}\right)$, equals to $4 \kappa_{a a}^{(1)}$.

In order to prove this, let us choose vector fields $\xi, \eta$ in $T^{C R} M$, a frame $u \in$ $\mathcal{G}, p(u)=x \in M$, and $X, Y \in \mathfrak{g}_{-1}$ such that $\xi(x)=T p \cdot \omega^{-1}(X)(u), \eta(x)=$ $T p \cdot \omega^{-1}(Y)(u)$. We have

$$
N(\xi(x), \eta(x))=[\xi, \eta]-[J \xi, J \eta]+J([J \xi, \eta]+[\xi, J \eta])
$$

and $N\left(T p \cdot \omega^{-1}(X)(u), T p \cdot \omega^{-1}(Y)(u)\right)=T p \cdot \omega^{-1}(\nu(u)(X, Y))$.

As before, there are projectable vector fields $\tilde{\xi}, \tilde{\eta}$ over $\xi$ and $\eta$, such that $\left[\omega^{-1}(X), \omega^{-1}(Y)\right](u)=[\tilde{\xi}, \tilde{\eta}](u)$ and similarly for $J \xi(x)=T p \cdot \omega^{-1}(i X)(u)$ and $J \eta(x)=T p \cdot \omega^{-1}(i Y)(u)$. Then we can compute

$$
\begin{aligned}
N(\xi(x), \eta(x))=T p \cdot\left(\left[\omega^{-1}(X), \omega^{-1}(Y)\right]-\left[\omega^{-1}(i X), \omega^{-1}(i Y)\right]+\right. & \\
& \left.\omega^{-1}\left(i \omega\left(\left[\omega^{-1}(i X), \omega^{-1}(Y)\right]+\left[\omega^{-1}(X), \omega^{-1}(i Y)\right]\right)(u)\right)\right)(u) \\
= & T p \cdot\left(\omega^{-1}([X, Y]-[i X, i Y]+i[i X, Y]+i[X, i Y]+\right. \\
& \left.\left.\kappa^{(1)}(X, Y)-\kappa^{(1)}(i X, i Y)+i \kappa^{(1)}(i X, Y)+i \kappa^{(1)}(X, i Y)\right)(u)\right)(u) \\
= & T p \cdot \omega^{-1}\left(4 \kappa_{a a}^{(1)}(u)(X, Y)\right)(u)
\end{aligned}
$$

3.7. The complexified Cartan connection. The proof of the preceding theorem could be also done by the methods of 3.4, with the help of complexification. Indeed, the complexification of the canonical form $\omega$ is $\omega_{\mathbb{C}}: T_{\mathbb{C}} \mathcal{G} \rightarrow \mathfrak{g}_{\mathbb{C}}$ which is a complex linear automorphism on each complex tangent space. The Lie bracket of real vector fields extends to the complex ones and again each choice of $u \in \mathcal{G}, X, Y \in$ $\left(\mathfrak{g}_{-}\right)_{\mathbb{C}}$ allows to choose projectable complex vector fields $\tilde{\xi}, \tilde{\eta}$ such that $[\tilde{\xi}, \tilde{\eta}](u)=$ $\left[\omega_{\mathbb{C}}^{-1}(X), \omega_{\mathbb{C}}^{-1}(Y)\right](u)$. Furthermore, the expansion of $\omega_{\mathbb{C}}\left(\left[\omega_{\mathbb{C}}^{-1}(X), \omega_{\mathbb{C}}^{-1}(Y)\right]\right)$ into the real and imaginary parts shows that the latter expression yields exactly the complexification $\kappa_{\mathbb{C}}$ of the curvature. Thus we may proceed exactly as in 3.5 in order to link the component of $\kappa_{\mathbb{C}}^{(1)}$ acting on two holomorphic vectors in the complexification of $\mathfrak{g}_{-1}$ 
and valued in the antiholomorphic ones, with the obstruction against the integrability of the holomorphic tangent subbundle in $\left(T^{C R} M\right)_{\mathbb{C}}$. Of course, the same applies if we swap the holomorphic and antiholomorphic vector fields.

3.8. The rest of the torsion. Similarly, the remaining two components of the curvature obtain a nice geometric interpretation in form of an algebraic bracket which is defined as follows. Take a holomorphic vector field $\xi \in\left(T^{C R, L} M\right)_{\mathbb{C}}$, an antiholomorphic $\eta \in\left(T^{C R, R} M\right)_{\mathbb{C}}$ and project their Lie bracket to the holomorphic component in $\left(T^{C R, R} M\right)_{\mathbb{C}}$. Clearly, this is an algebraic bracket and it vanishes if and only if the corresponding curvature component vanishes. Similarly to the involutivity of the holomorphic and antiholomorphic bundles, this obstruction has an tensorial interpretation $S_{R} \in\left(T^{C R, L} M\right)^{*} \otimes\left(T^{C R, R} M\right)^{*} \otimes T^{C R, R} M$,

$$
S_{R}(\xi, \eta)=\pi_{R}([\xi, \eta]+[J \xi, J \eta]-J[J \xi, \eta]+J[\xi, J \eta]) .
$$

Swapping the left and right tangent bundle components, we obtain

$$
S_{L} \in\left(T^{C R, R} M\right)^{*} \otimes\left(T^{C R, L} M\right)^{*} \otimes T^{C R, L} M .
$$

Theorem 3.9. Let $M \subset \mathbb{C}^{4}$ be a Levi non-degenerate 6-dimensional CR-manifold of CR-codimension 2 and let $x \in M$ be a hyperbolic point. Then $M$ is the product of two Levi non-degenerate 3-dimensional CR-structures $M_{1}, M_{2} \subset \mathbb{C}^{2}$, locally around $x$, if and only if the algebraic Lie brackets

$$
\begin{aligned}
& \{,\}_{L}: T^{L} M / T^{\mathrm{CR}, L} M \times T^{\mathrm{CR}, L} M \rightarrow T^{R} M / T^{\mathrm{CR}, R} M \\
& \{,\}_{R}: T^{R} M / T^{\mathrm{CR}, R} M \times T^{\mathrm{CR}, R} M \rightarrow T^{L} M / T^{\mathrm{CR}, L} M
\end{aligned}
$$

vanish on a neighborhood of $x$.

The abstract 6-dimensional hyperbolic CR-manifolds of CR-codimension two are locally products of (abstract) 3-dimensional $C R$-manifolds of $C R$-codimension 1 if and only if the above algebraic brackets, as well as the Nijenhuis tensor $N_{J} \in \Lambda^{2}\left(T^{C R} M\right)^{*} \otimes$ $T^{C R} M$ and tensors $S_{R}, S_{L}$ from (3.12), (3.13) vanish.

Proof. All considerations are local and so we may suppose that the whole $M$ is hyperbolic. If $M$ is a product of two 3-dimensional CR-manifolds, then we can also consider the product $\mathcal{G} \rightarrow M_{1} \times M_{2}$ of the corresponding canonical Cartan bundles $\mathcal{G}_{1} \rightarrow M_{1}, \mathcal{G}_{2} \rightarrow M_{2}$ equipped with the product $\omega=\omega_{1} \oplus \omega_{2}$ of the corresponding normal Cartan connections. These bundles and connections were constructed already by Cartan in [7] and their construction is also covered by Theorem 2.13. By definition, the new form $\omega \in \Omega^{1}(\mathcal{G}, \mathfrak{g})$ has all properties of normal Cartan connections and its curvature $\kappa$ is the sum of the two curvatures $\kappa_{1}$ and $\kappa_{2}$ of $\omega_{1}$ and $\omega_{2}$, respectively. In particular, there is no torsion because the connections $\omega_{1}$ and $\omega_{2}$ are torsion free. Thus the four tensorial obstructions on $M \subset \mathbb{C}^{4}$ have to vanish as well.

Now, let $M$ be an (abstract) hyperbolic 6-dimensional CR-manifold and assume that all six tensorial obstructions from our theorem vanish globally. According to previous results, all homogeneous components $\kappa^{(1)}$ of the torsion of the normal Cartan connection vanish globally. Thus, according to Theorem 2.8 and the table of the relevant cohomologies, all homogeneous components $\kappa^{(i)}, i \leq 3$, vanish too. In particular, there is no torsion part in $\kappa$. Let us consider next the part $\kappa^{L}$ of the whole curvature which is represented by cochains of the form $\mathfrak{g}_{-}^{L} \times \mathfrak{g}_{-}^{L} \rightarrow \mathfrak{g}^{R}$ and analogously $\kappa^{R}$ with left and right components swapped. We shall use the induction on the homogeneity 
degrees to show, that all these components vanish. Thus, assume we have done this for homogeneity less than $j$ and consider the components in homogeneity $j$. Since there are no cohomologies of the types in question, the corresponding parts of $\kappa^{L}$ and $\kappa^{R}$ are in the image of $\partial^{*}$ and so the differential $\partial$ acts on them injectively. Thus we can apply the Bianchi identity in order to see that there is no component which could contribute, cf. the end of the proof of Theorem 3.5. Consequently, both $\kappa^{L}$ and $\kappa^{R}$ vanish.

The splitting $\mathfrak{g}=\mathfrak{g}^{L} \oplus \mathfrak{g}^{R}$ induces two complementary $P$-invariant distributions on $\mathcal{G}, T \mathcal{G}=T^{L} \mathcal{G} \oplus T^{R} \mathcal{G}$. These distributions are involutive if and only if the obvious algebraic bracket $T^{L} \mathcal{G} \times T^{L} \mathcal{G} \rightarrow T^{R} \mathcal{G} \simeq T \mathcal{G} / T^{L} \mathcal{G}$ vanishes and similarly with $L$ and $R$ swapped. Since the brackets are algebraic, we may use the parallel fields $\omega^{-1}(X)$, $\omega^{-1}(Y)$ with properly chosen $X, Y$ for their evaluation. The projection may be realized by means of the component of $\omega$ valued in the left or right part of $\mathfrak{g}$. But this is controlled by the curvatures $\kappa^{L}, \kappa^{R}$ and so the brackets vanish, as proclaimed.

Now, we know that the Cartan bundle $\mathcal{G}$ locally splits into a product of two manifolds but we need much more. We wish to prove that there is a neighborhood of $x$ over which the whole Cartan bundle $(\mathcal{G}, \omega)$ is isomorphic to a product of $\left(\mathcal{G}_{L}, \alpha_{L}\right)$ and $\left(\mathcal{G}_{R}, \alpha_{R}\right)$ for some suitable Cartan connections $\alpha_{L}, \alpha_{R}$. In fact, if we construct these data only locally around a chosen frame $u \in \mathcal{G}$, then the right invariance will ensure what we need. The normal coordinates determined by the choice of $u$ will be again our basic tool.

So let $\varphi_{u}: \mathfrak{g}_{-}^{L} \oplus \mathfrak{g}_{-}^{R} \rightarrow \mathcal{G}$ be the mapping defined only locally around the origin by the horizontal flows and let $\sigma_{u}$ be the corresponding section of $\mathcal{G} \rightarrow M$. By abuse of notation, we shall not mention the definition domains of these and other locally defined mappings. Let us write $P_{L}$ and $P_{R}$ for the parabolic subgroups in the individual components of $G$ and define the trivial principal bundles $\mathcal{G}_{L}=\mathfrak{g}_{-}^{L} \times P_{L}, \mathcal{G}_{R}=\mathfrak{g}_{-}^{R} \times P_{R}$. Further, consider the principal fibre bundle morphism $\Psi: \mathcal{G}_{L} \times \mathcal{G}_{R} \rightarrow \mathcal{G}$ (notice $P_{L}$ and $P_{R}$ commute and the whole mapping is defined on fibers over a neighborhood of the origin in $\mathfrak{g}_{-}$only)

$$
\Psi:((X, p),(Y, q)) \mapsto \varphi_{u}(X, Y) p q .
$$

Furthermore, the restrictions of $\Psi$ yield principal fibre bundle morphisms

$$
\begin{aligned}
\Psi_{L}: \mathcal{G}_{L} \rightarrow \mathcal{G}, \quad(X, p) & \mapsto \varphi_{u}(X, 0) p \\
\Psi_{R}: \mathcal{G}_{R} \rightarrow \mathcal{G}, \quad(Y, q) & \mapsto \varphi_{u}(0, Y) q
\end{aligned}
$$

and consider the one forms $\alpha_{L}=\Psi_{L}^{*} \omega_{L}, \alpha_{R}=\Psi_{R}^{*} \omega_{R}$ where $\omega_{L}$ and $\omega_{R}$ are the left and right components of $\omega$. It remains to prove that $\left(\mathcal{G}_{L}, \alpha_{L}\right) \times\left(\mathcal{G}_{R}, \alpha_{R}\right)$ is a bundle with Cartan connection (defined locally over a neighborhood of the origin) and $\Psi^{*} \omega=\alpha_{L} \oplus \alpha_{R}$ wherever defined.

First notice that, due to our choices and the involutivity of the left and right parts of $T \mathcal{G}$, the forms $\alpha_{L}$ and $\alpha_{R}$ are pullbacks of the whole $\omega$ (viewed then as forms with values in $\mathfrak{g}$, but without any contribution to one half of the image). Thus the properties of the Cartan connections are simply transfered by $\Psi_{L}$ and $\Psi_{R}$. Furthermore, since the curvature of $\omega$ does not mix left and right sides either, the structure equations for $\alpha_{L}$ and $\alpha_{R}$ are obtained as pullbacks of the structure equation of $\omega$. In particular, the curvatures are again $\partial^{*}$ closed. Thus $\left(\mathcal{G}_{L}, \alpha_{L}\right)$ and $\left(\mathcal{G}_{R}, \alpha_{R}\right)$ are 3 -dimensional CRmanifolds of CR-codimension one (locally around the origin of the base manifolds). Finally, we observe that $\Psi^{*} \omega$ will (locally) coincide with the product of the newly constructed Cartan connections if and only if they will evaluate equally on vectors tangent 
to a fixed section of $\mathcal{G}_{L} \times \mathcal{G}_{R}$. Thus consider the section $(X, Y) \mapsto((X, e),(Y, e))$, evaluate $\left(\alpha_{L} \oplus \alpha_{R}\right)$ at the vector $(W, 0)+(0, Z) \in T_{((X, e),(Y, e))}\left(\mathcal{G}_{L} \times \mathcal{G}_{R}\right)$, and compare this with $\Psi^{*} \omega$. In fact, we may even deal with the left and right components of the tangent space separately.

Each such vector $\xi=\frac{\partial}{\partial t \mid 0}((X+t W, e),(Y, e))$ is mapped by $\Psi_{L}$ to $T\left(\Psi_{L}\right)(\xi)=$ $\frac{\partial}{\partial t \mid 0} \mathrm{Fl}_{1}^{X+t W}(u)$ and so we can easily compare the values $\alpha_{L}(\xi)$ and $\Psi^{*} \omega(\xi)$ :

$$
\begin{aligned}
& \left(\alpha_{L}\right)(\xi)=\omega_{L}\left(\frac{\partial}{\partial t} \mid 0 \mathrm{Fl}_{1}^{\omega^{-1}(X+t W)}(u)\right) \\
& \Psi^{*} \omega(\xi)=\omega_{L}\left(\frac{\partial}{\partial t} \mid 0 \mathrm{Fl}_{1}^{\omega^{-1}(X+t W+Y)}(u)\right) .
\end{aligned}
$$

Next, we observe that $\omega^{-1}(Y)$ commutes with $\omega^{-1}(X+t W)$ since there is no cohomology mixing the arguments from the left and right components of $\mathfrak{g}_{-}$. Thus we may rewrite the last expression as

$$
\begin{aligned}
\Psi^{*} \omega(\xi) & =\omega_{L}\left(T\left(\mathrm{Fl}_{1}^{\omega^{-1}(Y)}\right)\left(\left.\frac{\partial}{\partial t}\right|_{0} \mathrm{Fl}_{1}^{\omega^{-1}(X+t W)}(u)\right)\right) \\
& =\left(\mathrm{Fl}_{1}^{\omega^{-1}(Y)}\right)^{*} \omega_{L}\left(\frac{\partial}{\partial t \mid 0} \mathrm{Fl}_{1}^{\omega^{-1}(X+t W)}(u)\right) .
\end{aligned}
$$

Thus, in order to see that the two values coincide, it suffices to show that $\left(\mathrm{Fl}_{1}^{\omega^{-1}(Y)}\right)^{*} \omega_{L}=\omega_{L}$ for all $Y \in \mathfrak{g}_{-}^{R}$.

We know this for the flow in the time zero, $\mathrm{Fl}_{0}^{\omega^{-1}(Y)}=\mathrm{id}_{\mathcal{G}}$, and so we have just to show that $\frac{\partial}{\partial s}\left(\mathrm{~F}_{s}^{\omega^{-1}(Y)}\right)^{*} \omega_{L}$ vanishes identically. Each vector in the left component of $T_{v} \mathcal{G}$ is of the form $\omega^{-1}(V)(v)$ with $V \in \mathfrak{g}^{L}$ and we compute

$$
\begin{aligned}
\left(\mathrm{Fl}_{s}^{\omega^{-1}(Y)}\right)^{*} \omega_{L}\left(\frac{\partial}{\partial t} \mid 0 \mathrm{Fl}_{t}^{\omega^{-1}(V)}(v)\right) & =\omega_{L}\left(\frac{\partial}{\partial t} \mid 0 \mathrm{Fl}_{s}^{\omega^{-1}(Y)} \circ \mathrm{Fl}_{t}^{\omega^{-1}(V)}(v)\right) \\
& =\omega_{L}\left(\omega^{-1}(V)\left(\mathrm{Fl}_{s}^{\omega^{-1}(Y)}(v)\right)\right)=V .
\end{aligned}
$$

Since the derivative of this constant mapping vanishes, the required invariance of $\omega_{L}$ has been proved.

Similarly we deal with the other component $\omega_{R}$.

Finally we observe that if $M$ is embedded in $\mathbb{C}^{4}$, then we may always find embeddings $\phi_{i}$ of the components $M_{i}$ in neighborhoods of $x_{i}$ into $\mathbb{C}^{2}$ such that

$$
\phi=\phi_{1} \oplus \phi_{2}: M \rightarrow \mathbb{C}^{4}=\mathbb{C}^{2} \oplus \mathbb{C}^{2}
$$

is an embedding of $M$ at $x=\left(x_{1}, x_{2}\right)$. In fact, consider the initial embedding $\psi$ : $M \rightarrow \mathbb{C}^{4}$. Then the restriction of $\psi$ to $M_{1} \times\left\{x_{2}\right\}$ is an embedding of $M_{1}$ into $\mathbb{C}^{4}$ that respects the CR-structure of $M_{1}$. There is a holomorphic projection $\chi_{1}: \mathbb{C}^{4} \rightarrow \mathbb{C}^{2}$ that is diffeomorphic from $\psi\left(M_{1} \times\left\{x_{2}\right\}\right)$ onto its image. Denote the resulting mapping by $\phi_{1}$ and the analogous mapping for the second component by $\phi_{2}$. Then $\phi_{1} \oplus \phi_{2}$ is the desired embedding. By passing to normal forms (see Appendix B) one can even prove that the embeddings $\phi$ and $\psi$ are equivalent, i.e., $\phi=\Phi \circ \psi$ with some locally defined biholomorphic map $\Phi: \mathbb{C}^{4} \rightarrow \mathbb{C}^{4}$.

3.10. Chains. The last topic we want to discuss are the analogies to the chains on CR-manifolds of CR-codimension one. We have introduced the general concepts of chains and 1-chains in 2.4 for all parabolic geometries. These two notions coincide for the CR-manifolds of CR-codimension one and they also coincide with the chains defined in $[8]$. 
Let us recall that the amount of different 1-chains up to parameterizations passing in fixed direction through a given point $x \in M$, as well as the set of all chains through $x$ is visible from the homogeneous case (see 2.5).

The detailed discussion on the quadric $Q$ is reviewed in Appendix B with the following result: There is a one-parametric family of distinguished parameterizations on each (non-parametrized) 1-chain, and in each direction which does not belong to the subspace $T_{x}^{C R} M$ and does not belong to $T_{x}^{L} M$ neither to $T_{x}^{R} M$, there is a 1 parametric class of 1-chains up to their parameterizations, cf. (B.3). If the direction does belong to the left or right tangent space then there is a unique 1-chain in that direction. The chains through a given point $x \in M$ are available only in 2-dimensional directions of the form $\{u, X \wedge Y\} \in T_{x} M \wedge T_{x} M$ with $u \in \mathcal{G}$ in the fibre over $x$ and $X, Y \in \mathfrak{g}_{-2}$.

A general 2-dimensional surface is said to have the chain property at its point $y$ if there is a chain providing a parameterization of this surface around $y$.

The vector fields $\omega^{-1}(X), X \in \mathfrak{g}_{-2}$ span a two-dimensional distribution in $T \mathcal{G}$ which we call the chain distribution of the CR-structure.

In general, the two-dimensional (non-parameterized) chains $\beta^{u_{t}}$ rotate around one fixed 1-chain $\alpha^{u_{0}, X}(t)$ if we move the ruling frame $u_{t}=\mathrm{Fl}_{t}^{\omega^{-1}(X)}(u)$ along the horizontal flow. This is not possible, however, if the whole torsion of our CR-structure is zero, because then the whole chain distribution is integrable. This is in accordance with the previous theorem claiming that the whole bundle $\mathcal{G}$ is the product of two canonical Cartan bundles and the Cartan connection is a product, too. Thus, in the torsion-free case, our chains $\beta^{u}$ are obtained as products of the chains in the threedimensional CR-manifolds. In particular we have proved the following theorem.

THEOREM 3.11. Let $M \subset \mathbb{C}^{4}$ be an embedded 6-dimensional hyperbolic $C R$ manifold and assume that the algebraic brackets $\{,\}_{L}$ and $\{,\}_{R}$ from Theorem 3.9 vanish identically. Then each chain $\beta^{u}: U \subset \mathfrak{g}_{-2} \rightarrow M$ has the chain property at each of its points.

The same conclusion holds for abstract 6-dimensional hyperbolic CR-manifolds of CR-codimension 2 without torsion.

\section{The elliptic structures.}

4.1. Almost complex and almost product structures. Let us recall that on embedded elliptic 6-dimensional CR-manifolds of CR-codimension two, the fundamental osculation (1.1) provides the $(\mathfrak{g}, P)$ structure on $M$ with $\mathfrak{g}=\mathfrak{s l}(3, \mathbb{C})$, and its standard complex Borel subalgebra $\mathfrak{p}$ (both viewed as real Lie algebras). The proper choices for the groups $G, G_{0}, P$ are discussed in Appendix B.

There are striking general similarities between the hyperbolic and elliptic geometries. Indeed, the decomposition of the subspace $\mathfrak{g}_{-1} \subset \mathfrak{g}_{-}$

$$
\mathfrak{g}_{-1}=\mathfrak{g}_{-1}^{L} \oplus \mathfrak{g}_{-1}^{R}
$$

induces an almost product structure on the complex tangent bundle $T^{C R} M$. We shall write again $T^{C R, L} M$ and $T^{C R, R} M$ for the individual components. Furthermore, the complex structure of the whole real Lie algebra $\mathfrak{s l}(3, \mathbb{C})$ induces the almost complex structure $J$ on $T M$, given by the formula $J\left(T p \cdot \omega^{-1}(X)(u)\right)=T p \cdot \omega^{-1}(i X)(u)$. Clearly this formula is independent of the choice of $X$ and $u$ which give the same vector $T p \cdot \omega^{-1}(X)(u) \in T_{x} M$ because the adjoint action of $P$ on $\mathfrak{g}$ is complex linear. 


\begin{tabular}{|c|c|l|}
\hline homog. & cochains & comment \\
\hline 1 & $\mathfrak{g}_{-2} \times \mathfrak{g}_{-1}^{L} \rightarrow \mathfrak{g}_{-2}$ & antilinear in both arguments \\
1 & $\mathfrak{g}_{-2} \times \mathfrak{g}_{-1}^{R} \rightarrow \mathfrak{g}_{-2}$ & antilinear in both arguments \\
\hline 1 & $\mathfrak{g}_{-1}^{L} \times \mathfrak{g}_{-1}^{L} \rightarrow \mathfrak{g}_{-1}^{R}$ & totally real \\
1 & $\mathfrak{g}_{-1}^{R} \times \mathfrak{g}_{-1}^{R} \rightarrow \mathfrak{g}_{-1}^{L}$ & totally real \\
1 & $\mathfrak{g}_{-1}^{R} \times \mathfrak{g}_{-1}^{L} \rightarrow \mathfrak{g}_{-1}^{L}$ & sesquilinear \\
1 & $\mathfrak{g}_{-1}^{L} \times \mathfrak{g}_{-1}^{R} \rightarrow \mathfrak{g}_{-1}^{R}$ & sesquilinear \\
\hline 4 & $\mathfrak{g}_{-2} \times \mathfrak{g}_{-1}^{L} \rightarrow \mathfrak{g}_{1}^{L}$ & complex linear in both arguments \\
4 & $\mathfrak{g}_{-2} \times \mathfrak{g}_{-1}^{R} \rightarrow \mathfrak{g}_{1}^{R}$ & complex linear in both arguments \\
\hline
\end{tabular}

TABLE 4.1. Real second cohomologies of $\mathfrak{g}$ - with coefficients in $\mathfrak{g}=\mathfrak{s l}(3, \mathbb{C})$

As we have seen in the hyperbolic case, the knowledge of the real second cohomologies of the algebras in question is most essential. Also now, we shall mostly deal with the abstract $(\mathfrak{g}, P)$-structures defined on 6 -dimensional manifolds but we shall point out the specific properties of the embedded ones. In particular, all obstructions coming from cohomologies with cochains of the form $\mathfrak{g}_{-1} \times \mathfrak{g}_{-1} \rightarrow \mathfrak{g}_{-1}$ will disappear automatically according to Lemma 1.1.

Roughly speaking, the role of the integrability of the almost complex structures on the complex subbundles on hyperbolic manifolds is played by the integrability of the almost product structure on $T^{C R} M$ in the elliptic case. In particular the almost product structure will always be integrable on the embedded elliptic CR-manifolds. Further, the integrability of the almost product structure of the hyperbolic manifolds corresponds to the integrability of the almost complex structure $J$ on the elliptic ones. In particular, the almost complex structure $J$ is intrinsic to the manifold $M$ and it cannot be induced by the ambient complex structure in $\mathbb{C}^{4}$.

Lemma 4.2. All irreducible components in $H_{*}^{2}\left(\mathfrak{g}_{-}, \mathfrak{s l}(3, \mathbb{C})\right)$ are the one dimensional $G_{0}$-modules which are generated by the cochains listed in Table 4.1.

Proof. Exactly as in the hyperbolic case, the complexification of the cohomologies we want to describe is fully described by Table A.2 in Appendix A. Because of the complex structure on $\mathfrak{g}_{-}$, each of the real components will produce two copies in the complexification. In order to recognize them, we have to notice that complexifications of complex linear maps will not swap the two copies in the complexified Lie algebra, while the antilinear ones will swap them. This simple observation leads immediately to our Table 4.1.

THEOREM 4.3. The almost complex structure $J$ on an abstract elliptic CR-manifold of CR-codimension two is integrable if and only if the antilinear part $\kappa_{a a}^{(1)}$ of the curvature $\kappa$ of the canonical normal Cartan connection vanishes. This in turn happens if and only if the algebraic Lie brackets

$$
\begin{aligned}
& T^{(1,0)} M / T_{\mathbb{C}}^{C R} M \times\left(T^{C R, L} M\right)^{(1,0)} \rightarrow T^{(0,1)} M / T_{\mathbb{C}}^{C R} M \\
& T^{(1,0)} M / T_{\mathbb{C}}^{C R} M \times\left(T^{C R, R} M\right)^{(1,0)} \rightarrow T^{(0,1)} M / T_{\mathbb{C}}^{C R} M
\end{aligned}
$$


on the complexified graded tangent bundles vanish identically.

Proof. Essentially, all technique we need has been developed already. In particular, we may repeat the computation of the Nijenhuis tensor from the proof of Theorem 3.6. Since $\mathfrak{g}_{-}$is complex, we can do that with any $X, Y \in \mathfrak{g}_{-}$. The result tells us that the Nijenhuis tensor $N$, evaluated on $T p \cdot \omega^{-1}(X)(u), T p \cdot \omega^{-1}(Y)(u)$, is equal to

$$
T p \cdot \omega^{-1}\left(\left(4 \kappa_{a a}^{(1)}+4 \kappa_{a a}^{(2)}+4 \kappa_{a a}^{(3)}\right)(X, Y)(u)\right)(u) .
$$

Now, under the additional condition that the higher homogeneities cannot contribute whenever $\kappa_{a a}^{(1)}(X, Y)$ vanishes, the Nijenhuis tensor vanishes if and only if $\kappa_{a a}^{(1)}$ vanishes. According to the table of cohomologies, the latter expression must be given by the algebraic brackets (4.1), (4.2).

Thus we have to show, that if $\kappa_{a a}^{(1)}$ vanished, then no other antilinear component valued in $\mathfrak{g}_{-}$could occur in $\kappa^{(2)}$, and if so, than even not in $\kappa^{(3)}$. Let us assume the two brackets (4.1), (4.2) vanish. Then there is the algebraic bracket

$$
T^{(1,0)} M / T_{\mathbb{C}}^{C R} M \times\left(T^{C R, L} M\right)^{(1,0)} \rightarrow\left(T^{C R} M\right)^{(0,1)}
$$

which can be evaluated by means of the complexified curvature component of homogeneity two. Clearly this must come from an antilinear component and the vanishing of this algebraic bracket is equivalent to the vanishing of the antilinear parts $\kappa_{a a}^{(2)}: \mathfrak{g}_{-2} \times \mathfrak{g}_{-1}^{L} \rightarrow \mathfrak{g}_{-1}$. Using the Bianchi identity exactly as in the end of the proof of Lemma 3.4 we verify that there is no curvature like this.

Similarly we could proceed with the remaining algebraic brackets on the holomorphic tangent bundle with values in the antiholomorphic tangent bundle. However, the only component of homogeneity three is $\kappa_{a a}^{(3)}: \mathfrak{g}_{-2} \times \mathfrak{g}_{-2} \rightarrow \mathfrak{g}_{-1}$ and this vanishes automatically because it is complex antilinear and $\mathfrak{g}_{-2}$ is of (complex) dimension one.

THEOREM 4.4. Let $M$ be an abstract 6-dimensional elliptic CR-manifold with $C R$-codimension two. The distributions $T^{C R, L} M, T^{C R, R} M$ in the complex subspace $T^{C R} M$ are integrable if and only if the algebraic Lie brackets

$$
\begin{aligned}
& \left(T^{C R, L} M\right)^{(1,0)} \times\left(T^{C R, L} M\right)^{(0,1)} \rightarrow\left(T^{C R, R} M\right)^{(1,0)} \\
& \left(T^{C R, R} M\right)^{(1,0)} \times\left(T^{C R, R} M\right)^{(0,1)} \rightarrow\left(T^{C R, L} M\right)^{(1,0)}
\end{aligned}
$$

on the complexified complex spaces $T_{\mathbb{C}}^{C R} M$ vanish identically.

In particular, these almost product structures are always integrable on the embedded elliptic CR-manifolds.

Proof. The distributions are integrable if and only if the algebraic Lie brackets of two fields from the same component projected to the other one vanish. This is equivalent to the corresponding condition on the complexified bundles $T_{\mathbb{C}}^{C R}$. Now we may use the technique introduced in 3.7. Thus all the algebraic brackets in question will be linked to specific components of the curvature. Since they are all living on the CR tangent spaces, they must vanish automatically on the embedded elliptic manifolds.

On abstract manifolds, this means the brackets of holomorphic fields projected to the other component vanish automatically and the distribution $T^{C R, L} M$ is integrable if and only if the algebraic bracket (4.3) vanishes (cf. Table 4.1). The other distribution is treated similarly. 
4.5. Remaining torsion components. Let us notice that also the remaining two components of the torsion part of the curvature $\kappa$ of the canonical normal Cartan connection allow an expression by algebraic brackets. This time we obtain

$$
\begin{aligned}
& \left(T^{C R, R} M\right)^{(1,0)} \times\left(T^{C R, L} M\right)^{(0,1)} \rightarrow\left(T^{C R, L} M\right)^{(1,0)} \\
& \left(T^{C R, L} M\right)^{(1,0)} \times\left(T^{C R, R} M\right)^{(0,1)} \rightarrow\left(T^{C R, R} M\right)^{(1,0)}
\end{aligned}
$$

and they vanish again on all embedded elliptic 6-dimensional CR-manifolds in $\mathbb{C}^{4}$.

THEOREM 4.6. Let $M \subset \mathbb{C}^{4}$ be an embedded 6-dimensional elliptic CR-manifold of CR-codimension 2 and assume that the algebraic brackets (4.1), (4.2) both vanish. Then the complex structure $J$ on the entire Cartan bundle $\mathcal{G}$ is integrable, the normal Cartan connection is holomorphic, and there are two integrable foliations of $M$ by complex curves in $\mathbb{C}^{4}$ which span the complex subbundle $T^{C R} M$.

The same conclusion is true on the abstract 6-dimensional elliptic CR-manifolds if and only if all algebraic brackets (4.1), (4.2), (4.3), (4.4), (4.5), and (4.6) vanish identically.

Proof. In fact, we have nearly proved all necessary facts. Again, the same computation with the Nijenhuis tensor reveals, that the antilinear part $\kappa_{a a}$ of the entire curvature obstructs its integrability. Once we assume that all the torsion vanishes, there are no components of the curvature up to homogeneity four. This is not antilinear, however. A simple check with the Bianchi identity shows that the complex linear curvature components can never produce anything antilinear. Thus the integrability of the complex structure follows. Since the complex structure $J$ on $\mathcal{G}$ has been defined by the absolute parallelism $\omega$, clearly $\omega \in \Omega^{1}(\mathcal{G}, \mathfrak{g})$ is holomorphic.

On the abstract manifolds, the same argument applies if we assume that the whole torsion vanishes. On the other, each of the components of the torsion eventually produces some antilinear contribution in higher homogeneities via the Bianchi identity.

Now, assume $J$ is integrable and the torsion vanishes. Then also all horizontal vector fields $\omega^{-1}(X), \omega^{-1}(Y)$ with $X, Y \in \mathfrak{g}_{-1}^{L}$, or both in the other component, commute. Thus we obtain the integrable (real) 2-dimensional distributions in $T \mathcal{G}$ spanned by their values. The integral surfaces can be locally parameterized by the holomorphic (with respect to $J$ ) mappings

$$
\begin{aligned}
\gamma_{u}^{R}: \mathfrak{g}_{-1}^{R} \rightarrow T \mathcal{G}, & X \mapsto \mathrm{Fl}_{1}^{\omega^{-1}(X)}(u) \\
\gamma_{u}^{L}: \mathfrak{g}_{-1}^{L} \rightarrow T \mathcal{G}, & X \mapsto \mathrm{Fl}_{1}^{\omega^{-1}(X)}(u)
\end{aligned}
$$

and also their projections to $M$ will be holomorphic curves. Obviously, we have obtained integral manifolds for the distributions $T^{C R, R}$ and $T^{C R, L}$.

4.7. Chains. Let $M$ be a 6-dimensional elliptic CR-manifold with CR-codimension two, $x \in M, \xi \in T_{x} M$. As discussed in 2.4, the projections of the flows of horizontal vector fields determined by elements in $\mathfrak{g}_{-2}$ are 1-chains with specific properties, while

$$
\mathfrak{g}_{-2} \ni X \mapsto \mathrm{Fl}_{1}^{\omega^{-1}(X)}(u) \mapsto p\left(\mathrm{Fl}_{1}^{\omega^{-1}(X)}(u)\right)
$$

is the chain at $x$ determined by a fixed frame $u \in \mathcal{G}$ over $x$. A complex chain is a (locally defined) curve $\beta: \mathbb{C} \rightarrow M$ which is holomorphic with respect to the almost complex structure $J$ and has the chain property in all its points. 
THEOREM 4.8. If the brackets (4.1) and (4.2) vanish on a neighborhood of an elliptic point $x$ of an embedded 6-dimensional CR-manifold $M \subset \mathbb{C}^{4}$ of $C R$-codimension two, then there are unique complex chains through $x$ in all complex directions which do not belong to $T^{C R} M$.

The same conclusion is true for the abstract elliptic CR-manifolds if the other four obstructions against the vanishing of the torsion equal to zero too.

Proof. Analogously to the hyperbolic structures, there is the chain distribution in $T \mathcal{G}$ spanned by the horizontal fields $\omega^{-1}(X)$ with $X \in \mathfrak{g}_{-2}$. Again, the straightforward inspection of the possible curvature components reveals that there is no curvature with both arguments in $\mathfrak{g}_{-2}$ if the torsion vanishes. Thus the chain distribution is integrable. Consequently the flows of the horizontal fields yield holomorphic parameterizations and the theorem is proved.

\section{Final remarks and conclusions.}

5.1. Relation to other results. Mizner [17] constructed CR-invariant connections for weakly uniform CR-structures of codimension 2. In the cases considered there, the automorphisms of the quadrics are always linear (thus, $\mathfrak{p}_{+}$is absent) and so [17] does not come even close to our results in the distinguished 6-dimensional case. Similar results were obtained by Garrity and Mizner for CR-structures of codimension bigger than 2 with rigid osculating quadrics. The CR-manifolds that are considered in this paper are not covered there.

In [9] Ezhov, Isaev and Schmalz constructed parallelisms for hyperbolic and elliptic manifolds. These parallelisms turn out to be Cartan connections only in very special cases. The geometric reason for that is the presence of torsion in our Car$\tan$ connection. We were able to characterize the torsion-free ("semi-flat") cases as manifolds with integrable almost product structure in the hyperbolic case and with integrable almost complex structure in the elliptic case. Thus we give an answer to the question about the geometric meaning of "semi-flatness" for elliptic manifolds that has been posed in [9].

Let us also remark that the almost CR-manifolds of CR-codimension one (e.g. certain real hypersurfaces in almost complex manifolds) have been studied from the point of view of the general theory of parabolic geometries in [3]. In particular, a nice geometric specification of the construction from 2.13 is presented there.

5.2. The parabolic CR-geometry. Unfortunately, the automorphism group of the parabolic quadric (1.3) does not fit into our scheme of general parabolic geometries at all (notice the abuse of the non-compatible use of the word "parabolic" which is used in the sense of Section 2 now). This is obvious already from its dimension which is 17. The structure of its infinitesimal automorphisms is described in detail in [19] and it turns out that the discrete center $\mathbb{Z}_{2}$ of the hyperbolic or elliptic group blows up into the additional dimension and one copy of $\mathfrak{s u}(2,1)$ sits still inside. So it plays nicely its role of an intermediate state between the hyperbolic and elliptic points.

In particular the methods of Section 2 which are based on the existence of the Hodge theory on the cochains in the Lie algebra cohomology cannot work. One should believe that some specification of the very general approach in [18] could be applicable. We consider this as a very interesting open problem.

5.3. Webster-Tanaka connections. There is a very rich underlying geometry on each manifold equipped with a Cartan connection modeled over graded Lie algebras. In particular, we always have the principal bundles $\mathcal{G}_{0}=\mathcal{G} / P_{+} \rightarrow M$ with 
structure group $G_{0}$ and the principal bundle $\mathcal{G} \rightarrow \mathcal{G}_{0}$ with the structure group $P_{+}$. The latter bundle always admits global smooth $G_{0}$-equivariant sections and the set of all of them is parameterized by one-forms on $M$. The pullback of the $\left(\mathfrak{g}_{-} \oplus \mathfrak{g}_{0}\right)$ component of the Cartan connection $\omega$ by means of any of these sections provides an affine connection on $T M$, i.e. a soldering form on $\mathcal{G}_{0}$ together with a principal connection on $\mathcal{G}_{0}$. This construction has been described in full generality in [21] and it produces the Webster-Tanaka connections on the CR-manifolds with CR-codimension one. Thus we have a similar class of linear connections on $M$ underlying our elliptic and hyperbolic structures.

5.4. Natural bundles and invariant operators. Another very interesting consequence of our construction of the canonical Cartan connections is the theory of the semi-holonomic jet modules for general parabolic geometries, which allows to transfer the problem of finding invariant operators which act on some natural bundles coming from representations of $P$ into problems in finite dimensional representation theory. The first application of this theory is worked out in $[5,6]$.

In particular, there are the Bernstein-Gelfand-Gelfand sequences for all irreducible $G$-modules $\mathbb{V}$ which specialize to the BGG resolution of the constant sheaf with coefficients in $\mathbb{V}$ on the homogeneous space, see [6]. The analogies to classical complexes on CR-manifolds with CR-codimension one should be localized inside of these sequences.

A. Cohomologies. The aim of this section is to provide the list of all non-zero cohomologies in $H^{2}\left(\mathfrak{g}_{-}, \mathfrak{g}\right)$ for the complex algebras

$$
\mathfrak{g}=\mathfrak{s l}(3, \mathbb{C}) \oplus \mathfrak{s l}(3, \mathbb{C}) \quad \mathfrak{p}=\{\text { all upper triangular matrices in } \mathfrak{g}\}
$$

We shall refer to the two copies of $\mathfrak{s l}(3, \mathbb{C})$ as the left and right ones. The two parts of $\mathfrak{g}_{0}$ coincide with the parts of the Cartan subalgebra of the diagonal matrices and all the one-dimensional root spaces are (complex) one-dimensional. We shall denote them as indicated in the following matrices

$$
\mathfrak{g}=\left(\begin{array}{ccc}
* & \mathfrak{g}_{1,0}^{L} & \mathfrak{g}_{2}^{L} \\
\mathfrak{g}_{-1,0}^{L} & * & \mathfrak{g}_{0,1}^{L} \\
\mathfrak{g}_{-2}^{L} & \mathfrak{g}_{0,-1}^{L} & *
\end{array}\right) \oplus\left(\begin{array}{ccc}
* & \mathfrak{g}_{1,0}^{R} & \mathfrak{g}_{2}^{R} \\
\mathfrak{g}_{-1,0}^{R} & * & \mathfrak{g}_{0,1}^{R} \\
\mathfrak{g}_{-2}^{R} & \mathfrak{g}_{0,-1}^{R} & *
\end{array}\right)
$$

Here the stars fill up the subalgebra $\mathfrak{g}_{0}, \mathfrak{p}_{+}$consists of the strictly upper triangular matrices, $\mathfrak{g}_{1}=\mathfrak{g}_{1,0} \oplus \mathfrak{g}_{0,1}$ as $\mathfrak{g}_{0}$-module, etc.

The cohomologies for modules over simple algebras are completely described in terms of the orbits of the Weyl groups on the weights. We shall use the notation and technique as developed in [2]. First, we have to recall a few basic facts on the representations of the parabolic subalgebra $\mathfrak{p} \subset \mathfrak{g}$.

The Dynkin diagram of $\mathfrak{s l}(3, \mathbb{C})$ is $\bullet$. The parabolic subalgebras are denoted by crossing the nodes which correspond to the negative simple coroots which do not belong to $\mathfrak{p}$. In our case this means one $\longleftrightarrow$ for both left and right $\mathfrak{s l}(3, \mathbb{C})$. The weights of irreducible representations of $\mathfrak{p}$ are then denoted by the coefficients in their expressions as linear combinations of fundamental weights, placed over the corresponding nodes. The $\mathfrak{g}$-dominant weights have non-negative integral coefficients, the $\mathfrak{p}$-dominant weights must be non-negative over the uncrossed nodes only. For example, the trivial representation and the first and second fundamental representations of $\mathfrak{s l}(3, \mathbb{C})$ have the highest weights $\stackrel{0}{x} \stackrel{0}{x}, \stackrel{1}{\longleftarrow} \stackrel{0}{\longleftarrow}, \stackrel{0}{\times} \stackrel{1}{x}$. Each $\mathfrak{p}$-module enjoys the filtration of the $\mathfrak{p}$-submodules such that the associated graded $\mathfrak{p}$ module decomposes 
into the direct sum of irreducible $\mathfrak{p}$-modules. For example, the filtration and decomposition of the $\mathfrak{p}$-module $\mathfrak{s l}(3, \mathbb{C})$ is as follows

$$
\mathfrak{g}_{-2}+\underset{\mathfrak{g}_{0,-1}}{\oplus}+\mathfrak{g}_{0}+\underset{\mathfrak{g}_{0,1}}{\oplus}+\underset{\mathfrak{g}_{1,0}}{\oplus}+\mathfrak{g}_{2}
$$

and in the terms of the highest weights for the one-dimensional irreducible $\mathfrak{p}$-modules

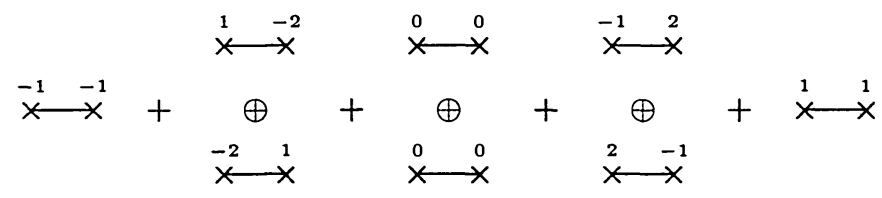

The whole Weyl group $W$ of $\mathfrak{s l}(3, \mathbb{C})$ is generated by the two simple reflections $s_{1}, s_{2}$ with respect to the two simple roots, acting on the weights $\mathfrak{g}_{0}^{*}$.

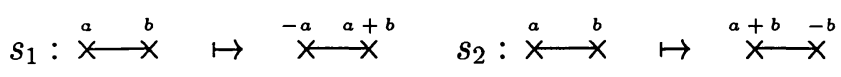

Since our parabolic subalgebra $\mathfrak{p} \subset \mathfrak{g}$ is the Borel subalgebra, the corresponding parabolic subgroup $W^{\mathfrak{p}}$ coincides with the whole $W$.

The differential $\partial$ respects the homogeneities of the cochains and so the cohomologies split into homogeneous components $H_{\ell}^{*}\left(\mathfrak{g}_{-}, \mathfrak{g}\right)$ too. Moreover, we have the identification $H_{\ell}^{p}\left(\mathfrak{g}_{-}, \mathfrak{g}\right) \simeq H_{-\ell}^{p}\left(\mathfrak{p}_{+}, \mathfrak{g}\right)$ (of real vector spaces). Thus the Kostant's version of the Bott-Borel-Weil theorem is relevant for our aims as well:

THEOREM A.1. Let $\mathfrak{p} \subset \mathfrak{g}$ be a parabolic subalgebra in a complex simple algebra $\mathfrak{g}$. If $A$ is a finite dimensional irreducible $\mathfrak{g}$-module of highest weight $\lambda$, then the whole cohomology $H^{*}\left(\mathfrak{p}_{+}, A\right)$ is completely reducible as a $\mathfrak{p}$-module and the irreducible components with highest weight $\mu$ occur if and only if there is an element $w \in W^{\mathfrak{p}} \subset W$ such that $\mu=w \cdot \lambda=w(\lambda+\rho)-\rho$ and in that case it occurs in degree $|w|$ with multiplicity one.

The degree of an element $w \in W$ is defined as the smallest possible number of simple reflections whose composition is $w$. See e.g. $[15,25]$ for the proof the Theorem.

Now, we have a simple procedure to compute the cohomologies: First, we write down the labeled Dynkin diagram depicting the $\mathfrak{g}$-dominant highest weight $\lambda$. For example, the highest weight of the adjoint representation is $\stackrel{1}{\longleftarrow} \stackrel{1}{x}$. Then we add one to each coefficient and act by combinations of simple reflections according to (A.4). Finally we subtract one from each coefficient. The $\mathfrak{p}$-dominant results are just the highest weights of the cohomologies.

Unfortunately, we deal with a sum $\mathfrak{g}=\mathfrak{g}^{L} \oplus \mathfrak{g}^{R}$ of two simple algebras. In order to make use of the latter theorem, we shall view the representation spaces of $\mathfrak{g}$ and $\mathfrak{p}$ as the (exterior) tensor products $A \otimes B$ of $\mathfrak{g}^{L}$-modules $A$ and $\mathfrak{g}^{R}$-modules $B$. In particular, we understand the adjoint representation on $\mathfrak{g}=\mathfrak{g}^{L} \oplus \mathfrak{g}^{R}$ as

$$
\mathfrak{g}=\left(\mathfrak{g}^{L} \otimes \mathbb{C}\right) \oplus\left(\mathbb{C} \otimes \mathfrak{g}^{R}\right)
$$

with the obvious tensorial actions of $\mathfrak{p}_{+}=\mathfrak{p}_{+}^{L} \oplus \mathfrak{p}_{+}^{R}$. 


\begin{tabular}{|c|c|c|c|}
\hline$H^{0}\left(\mathfrak{p}_{+}, \mathbb{C}\right)$ & $\stackrel{0}{\longleftarrow} \stackrel{0}{x}$ & $H^{0}\left(\mathfrak{p}_{+}, \mathfrak{s l}(3, \mathbb{C})\right)$ & $\stackrel{1}{x} \stackrel{1}{x}$ \\
\hline$H^{1}\left(\mathfrak{p}_{+}, \mathbb{C}\right)$ & $\begin{array}{ll}\dot{x}^{2} & 1 \\
\longleftrightarrow & x \\
1 & -2 \\
x & x\end{array}$ & $H^{1}\left(\mathfrak{p}_{+}, \mathfrak{s l}(3, \mathbb{C})\right)$ & $\begin{array}{cc}-3 & 3 \\
\times & x \\
3 & -3 \\
x & x\end{array}$ \\
\hline$H^{2}\left(\mathfrak{p}_{+}, \mathbb{C}\right)$ & $\begin{array}{ll}0 & -3 \\
\times & \times \\
-3 & 0 \\
\times & \times\end{array}$ & $H^{2}\left(\mathfrak{p}_{+}, \mathfrak{s l}(3, \mathbb{C})\right)$ & $\begin{array}{ll}1 & -5 \\
\longleftrightarrow & \times \\
-5 & 1 \\
\check{x} & \end{array}$ \\
\hline
\end{tabular}

TABLE A.1.

The cohomology with values in a direct sum of modules is just the direct sum of the cohomologies with values in the submodules. Now, the Künneth theorem implies for each tensor product of our modules $A \otimes B$

$$
H^{p}\left(\mathfrak{p}_{+}^{L} \oplus \mathfrak{p}_{+}^{R}, A \otimes B\right)=\sum_{i+j=p}\left(H^{i}\left(\mathfrak{p}_{+}^{L}, A\right) \otimes H^{j}\left(\mathfrak{p}_{+}^{R}, B\right)\right)
$$

Thus, in order to compute the second cohomologies

$$
H_{\ell}^{2}\left(\mathfrak{p}_{+}^{L} \oplus \mathfrak{p}_{+}^{R}, \mathfrak{s l}(3, \mathbb{C}) \oplus \mathfrak{s l}(3, \mathbb{C})\right)
$$

we have to know all cohomologies $H_{*}^{i}\left(\mathfrak{p}_{+}, \mathfrak{s l}(3, \mathbb{C})\right), i=0,1,2$, and $H_{*}^{i}\left(\mathfrak{p}_{+}, \mathbb{C}\right)$. The results computed by the procedure as described above are listed in Table A.1.

The homogeneity of the components is given by the sum of the coefficients, which is the action by the so called grading element $E \in \mathfrak{s l}(3, \mathbb{C}), E=\operatorname{diag}(1,0,-1)$. There is another independent element $F \in \mathfrak{s l}(3, \mathbb{C}), F=\operatorname{diag}(1,-2 / 3,1)$, which acts trivially on $\mathfrak{g}_{2}$, by 1 on $\mathfrak{g}_{-1,0}$, and by -1 on $\mathfrak{g}_{0,-1}$. Thus the action of $F$ on a weight module is given by one third of the difference of the coefficients over the nodes in the Dynkin diagram.

Now, the rest of our computation is quite easy since all irreducible components in the cohomologies are one-dimensional. Thus in order to localize the representatives of cohomologies as bilinear mappings, we have just to evaluate the actions of the left and right $g_{0}$-elements $E^{L}, E^{R}, F^{L}, F^{R}$ on the weight modules in the second cohomologies and this always describes the possible domain and target of a bilinear representative in the space of cochains uniquely. A half of the result is listed in Table A.2. The other half is obtained by mutually replacing all the left and right components.

In fact, we are interested in the real cohomologies $H_{\ell}^{2}\left(\mathfrak{g}_{-}, \mathfrak{s u}(2,1) \oplus \mathfrak{s u}(2,1)\right)$ and $H_{\ell}^{2}\left(\mathfrak{g}_{-}, \mathfrak{s l}(3, \mathbb{C})\right)$ where $\mathfrak{g}_{-}$is the negative complement to the real Borel subalgebras $\mathfrak{p}$. As we have mentioned already, the latter cohomologies are dual to the real cohomologies $H_{-\ell}^{2}\left(\mathfrak{p}_{+}, \mathfrak{s u}(2,1) \oplus \mathfrak{s u}(2,1)\right)$ and $H_{-\ell}^{2}\left(\mathfrak{g}_{-}, \mathfrak{s l}(3, \mathbb{C})\right)$. Thus the complexifications of the requested cohomologies will be dual (as real modules) to those listed in Table A.2.

B. Normal forms. For embedded real-analytic hyperbolic or elliptic CR-manifolds one has constructions of normal coordinates in the ambient space in a neighborhood of a given point. These coordinates are uniquely determined up to some Lie-group action of the isotropy group of the quadric (1.2) resp. (1.4). The equation of the manifold takes then a special form called normal form that refines the osculation (1.1) by the quadric. These constructions generalize Chern-Moser's normal form for real-analytic hypersurfaces in $\mathbb{C}^{n}$. They were obtained by Loboda [16] in the hyperbolic and by Ezhov and Schmalz [11] in the elliptic case. 


\begin{tabular}{|c|c|c|c|}
\hline \multicolumn{2}{|c|}{ homogeneities } & \multicolumn{2}{|c|}{ components in $H^{2}\left(\mathfrak{p}_{+}^{L} \oplus \mathfrak{p}_{+}^{R}, \mathfrak{g}^{L} \otimes \mathbb{C}\right)$} \\
\hline total & $\begin{array}{c}\text { actions of } \\
E^{L}, E^{R}, F^{L}, F^{R}\end{array}$ & components & cochains \\
\hline-1 & $2,-3,0,1$ & $\stackrel{1}{\times} \stackrel{1}{x} \otimes \stackrel{0}{x} \stackrel{-3}{x}$ & $\mathfrak{g}_{2}^{R} \times \mathfrak{g}_{1,0}^{R} \rightarrow \mathfrak{g}_{2}^{L}$ \\
\hline-1 & $2,-3,0,-1$ & $\stackrel{1}{\longleftarrow} \stackrel{1}{\times} \otimes \stackrel{-3}{\leftarrow} \stackrel{0}{\times}$ & $\mathfrak{g}_{2}^{R} \times \mathfrak{g}_{0,1}^{R} \rightarrow \mathfrak{g}_{2}^{L}$ \\
\hline-1 & $0,-1,-2,-1$ & $\stackrel{-3}{\leftarrow} \stackrel{3}{\leftarrow} \otimes \stackrel{-2}{x} \stackrel{1}{x}$ & $\mathfrak{g}_{0,1}^{L} \times \mathfrak{g}_{0,1}^{R} \rightarrow \mathfrak{g}_{1,0}^{L}$ \\
\hline-1 & $0,-1,-2,1$ & $\stackrel{-3}{x} \stackrel{3}{x} \otimes \stackrel{1}{x} \stackrel{-2}{x}$ & $\mathfrak{g}_{0,1}^{L} \times \mathfrak{g}_{1,0}^{R} \rightarrow \mathfrak{g}_{1,0}^{L}$ \\
\hline-1 & $0,-1,2,-1$ & $\stackrel{3}{\longleftarrow} \stackrel{-3}{x} \Delta \stackrel{-2}{x} \stackrel{1}{x}$ & $\mathfrak{g}_{1,0}^{L} \times \mathfrak{g}_{0,1}^{R} \rightarrow \mathfrak{g}_{0,1}^{L}$ \\
\hline-1 & $0,-1,2,1$ & $\stackrel{3}{\times} \stackrel{-3}{x} \otimes \stackrel{1}{\times} \quad{ }^{-2} \times$ & $\mathfrak{g}_{1,0}^{L} \times \mathfrak{g}_{1,0}^{R} \rightarrow \mathfrak{g}_{0,1}^{L}$ \\
\hline-4 & $-4,2,0,0$ & $\stackrel{1}{\times} \stackrel{-5}{x} \otimes \stackrel{0}{x} \stackrel{0}{\leftarrow}$ & $\mathfrak{g}_{2}^{L} \times \mathfrak{g}_{1,0}^{L} \rightarrow \mathfrak{g}_{-1,0}^{L}$ \\
\hline-4 & $-4,-2,0,0$ & $\stackrel{-5}{\times} \stackrel{1}{\times} \otimes \stackrel{0}{\times} \stackrel{0}{\leftarrow}$ & $\mathfrak{g}_{2}^{L} \times \mathfrak{g}_{0,1}^{L} \rightarrow \mathfrak{g}_{0,-1}^{L}$ \\
\hline
\end{tabular}

TABle A.2.

Let us recall the isotropy groups of the quadrics. It is convenient to choose coordinates that reflect the geometric structure of the quadrics. The hyperbolic quadric is the direct product of two hyperspheres in $\mathbb{C}^{2}$ :

$$
v_{1}=\left|z_{1}\right|^{2}, \quad v_{2}=\left|z_{2}\right|^{2} \text {. }
$$

The geometric structure of the elliptic quadric will be revealed by passing to coordinates

$$
w_{1}^{\sharp}=w_{1}+i w_{2}, \quad w_{2}^{\sharp}=w_{1}-i w_{2} .
$$

Then $\mathcal{V}=v_{1}+i v_{2}=\frac{w_{1}^{\sharp}-\bar{w}_{2}^{\sharp}}{2 i}$ and the equation of the quadric takes the form

$$
\mathcal{V}=\frac{w_{1}^{\sharp}-\bar{w}_{2}^{\sharp}}{2 i}=z_{1} \bar{z}_{2} .
$$

Thus this quadric carries a complex structure; it is a complex hypersurface in $\mathbb{C}^{4}$ with coordinates $z_{1}, \bar{z}_{2}, w_{1}^{\sharp}, \bar{w}_{2}^{\sharp}$. Below we will use these coordinates and omit the sharps.

The automorphism group of any quadric contains a transitive subgroup called Heisenberg group. For any point $(p, q)$ at the quadric the Heisenberg translation that takes the origin into $(p, q)$ has the form

$$
\begin{aligned}
z^{*} & =z+p \\
w^{*} & =w+q+2 i\langle z, p\rangle .
\end{aligned}
$$

Thus, any automorphism decomposes into a Heisenberg translation and an isotropic automorphism. The subgroup of isotropic automorphisms will play the role of the parabolic subgroup $P$ with Lie algebra $\mathfrak{p}$.

For both our quadrics the isotropic automorphisms can be described by the wellknown Poincaré formula for sphere automorphisms

$$
\begin{aligned}
Z^{*} & =C(Z+A W)(1-2 i \bar{A} Z-(R+i A \bar{A}) W)^{-1} \\
W^{*} & =C \bar{C} W(1-2 i \bar{A} Z-(R+i A \bar{A}) W)^{-1}
\end{aligned}
$$


where $Z$ and $W$ are diagonal $2 \times 2$-matrices with entries $z_{1}, z_{2}$ and $w_{1}, w_{2}$, respectively, $C$ and $A$ are complex diagonal matrices, and $R$ is a real diagonal matrix. There occurs an additional discrete automorphism that interchanges $z_{1} \leftrightarrow z_{2}$ and $w_{1} \leftrightarrow w_{2}$. The group $P$ decomposes into $G_{0}$ and $P_{+}$where $G_{0}$ consists of the linear isotropic automorphisms (with $A=R=0$, including the discrete automorphism) and $P_{+}$ consists of the "non-linear" automorphisms with $C=1$. The Lie algebras of the latter subgroups are $\mathfrak{g}_{0}$ and $\mathfrak{p}_{+}$, respectively.

The only difference between the hyperbolic and elliptic case is a different definition of the complex conjugation. In the hyperbolic case the conjugation is the usual one and $R$ is real means that it has real entries. Thus, the automorphisms also split into a direct product. Since the automorphism group of the sphere is $\mathrm{SU}(2,1) / \mathbb{Z}_{3}$ this shows that the automorphism group of the hyperbolic quadric is

$$
\left(\left(\mathrm{SU}(2,1) / \mathbb{Z}_{3}\right) \times\left(\mathrm{SU}(2,1) / \mathbb{Z}_{3}\right)\right) \rtimes \mathbb{Z}_{2}
$$

In the elliptic case the complex conjugation is the usual one combined with interchanging $z_{1} \leftrightarrow z_{2}$ and $w_{1} \leftrightarrow w_{2} . R$ is real means now that the entries are mutually complex conjugate numbers. The identification of the automorphism group $G$ as

$$
\left(\mathrm{SL}(3, \mathbb{C}) / \mathbb{Z}_{3}\right) \rtimes \mathbb{Z}_{2}
$$

is less evident than in the hyperbolic case. As shown in [19] the Lie algebra of infinitesimal automorphisms of the elliptic quadric is isomorphic to $\mathfrak{s l}(3, \mathbb{C})$. Since $G$ acts effectively at $\mathfrak{s l}(3, \mathbb{C})$ via Ad one can consider $G$ as a subgroup of Aut $\mathfrak{s l}(3, \mathbb{C})$. Both groups have the same dimension and consist of two connected components. Therefore they must coincide. It is not hard to check that Aut $\mathfrak{s l}(3, \mathbb{C}) \cong \operatorname{SL}(3, \mathbb{C}) / \mathbb{Z}_{3} \rtimes \mathbb{Z}_{2}$.

It follows from the explicit description of the action of $G$ on the infinitesimal automorphisms that $P$ is exactly the subgroup that respects the filtration of $\mathfrak{g}$ by the p-submodules (cf. 2.1).

Let us remark that the hyperbolic and elliptic quadrics have compact completions in $\mathbb{C P}^{2} \times \mathbb{C P}^{2}$ resp. $\mathbb{C P}^{2} \otimes \mathbb{C}$. All automorphisms extend to automorphisms of the completion and are then linear with respect to the corresponding homogeneous coordinates (see [10]). Moreover, these completions can be considered as $G / P$.

Now we formulate the concrete normal form conditions: In the hyperbolic case the normalized equation of the manifolds takes the form

$$
v_{j}=\left|z_{j}\right|^{2}+\sum N_{k l}^{j}(z, \bar{z}, u)
$$

where $N_{k l}^{j}=\overline{N_{l k}^{j}}$ are polynomials of degree $k$ in $z$ and of degree $l$ in $\bar{z}$ with coefficients that are analytic functions of $u=\operatorname{Re} w$. The summation runs over all integral $k, l$ 
with $\max \{k, l\}>1$ and $\min \{k, l\}>0$ The polynomials satisfy the conditions

$$
\begin{array}{rlrl}
\frac{\partial N_{k 1}^{1}}{\partial \bar{z}_{1}} & =0 & \frac{\partial N_{k 1}^{2}}{\partial \bar{z}_{2}} & =0, \quad \text { for } k \geq 2 \\
\frac{\partial^{2} N_{21}^{1}}{\partial z_{1} \partial z_{2}} & =0 & \frac{\partial^{2} N_{21}^{2}}{\partial z_{1} \partial z_{2}}=0 \\
\frac{\partial^{4} N_{22}^{1}}{\partial z_{1} \partial z_{2} \partial \bar{z}_{1} \partial \bar{z}_{2}}=0 & \frac{\partial^{4} N_{22}^{2}}{\partial z_{1} \partial z_{2} \partial \bar{z}_{1} \partial \bar{z}_{2}}=0 \\
\frac{\partial^{4} N_{22}^{1}}{\left(\partial z_{1}\right)^{2}\left(\partial \bar{z}_{1}\right)^{2}}{ }_{\mid u_{2}=0}=0 & \frac{\partial^{4} N_{22}^{2}}{\left(\partial z_{2}\right)^{2}\left(\partial \bar{z}_{2}\right)^{2}}{ }_{\mid u_{1}=0}=0 \\
\frac{\partial^{5} N_{32}^{1}}{\left(\partial z_{1}\right)^{3}\left(\partial \bar{z}_{1}\right)^{2}}{ }_{\mid u_{2}=0}=0 & \frac{\partial^{5} N_{32}^{2}}{\left(\partial z_{2}\right)^{3}\left(\partial \bar{z}_{2}\right)^{2}}{ }_{\mid u_{1}=0}=0 \\
\frac{\partial^{6} N_{33}^{1}}{\left(\partial z_{1}\right)^{3}\left(\partial \bar{z}_{1}\right)^{3}}{ }_{\mid u_{2}=0}=0 & \frac{\partial^{6} N_{33}^{2}}{\left(\partial z_{2}\right)^{3}\left(\partial \bar{z}_{2}\right)^{3}}{ }_{\mid u_{1}=0}=0 .
\end{array}
$$

The manifold $M$ is torsion-free if and only if $N^{j}$ depends only on $z_{j}, \bar{z}_{j}$ and $u_{j}$. Then the conditions above coincide with Chern Moser's conditions.

In the elliptic case the normalized equation takes the form

$$
\mathcal{V}=z_{1} \bar{z}_{2}+\sum N_{k l}(z, \bar{z}, \mathcal{U}, \overline{\mathcal{U}})
$$

where $\mathcal{U}=\frac{w_{1}+\bar{w}_{2}}{2}$ and the $N_{k l}$ are polynomials as above (though without additional reality condition). The summation is also as above and the polynomials satisfy the conditions

$$
\begin{array}{rlrl}
\frac{\partial N_{k 1}}{\partial \bar{z}_{2}} & =0 & \frac{\partial N_{1 k}}{\partial z_{1}} & =0, \text { for } k \geq 2 \\
\frac{\partial^{3} N_{21}}{\partial z_{1} \partial \bar{z}_{1} \partial z_{2}} & =0 & \frac{\partial^{3} N_{12}}{\partial \bar{z}_{2} \partial \bar{z}_{1} \partial z_{2}} & =0 \\
\frac{\partial^{4} N_{22}}{\partial z_{1} \partial z_{2} \partial \bar{z}_{1} \partial \bar{z}_{2}} & =0 & \frac{\partial^{4} N_{22}}{\left(\partial z_{1}\right)^{2}\left(\partial \bar{z}_{2}\right)^{2}}{ }_{\mid \bar{\eta}=0}=0 \\
\frac{\partial^{5} N_{32}}{\left(\partial z_{1}\right)^{3}\left(\partial \bar{z}_{2}\right)^{2}}{ }_{\mid \bar{\eta}=0}=0 & \frac{\partial^{5} N_{23}}{\left(\partial z_{1}\right)^{2}\left(\partial \bar{z}_{2}\right)^{3}}{ }_{\mid \bar{\eta}=0}=0 \\
\frac{\partial^{6} N_{33}}{\left(\partial z_{1}\right)^{3}\left(\partial \bar{z}_{2}\right)^{3}}{ }_{\mid \bar{\eta}=0}=0 . &
\end{array}
$$

Elliptic torsion-free manifolds are obtained for $N=N\left(z_{1}, \bar{z}_{2}, \mathcal{U}\right)$. Then $M$ is a complex hypersurface in $\mathbb{C}^{4}$ with complex coordinates $\left(z_{1}, \bar{z}_{2}, w_{1}, \bar{w}_{2}\right)$.

From the normal form one can see that the real 2-dimensional surface $\{z=0, v=$ $0\}$ resp. $\{z=0, \mathcal{V}=0\}$ is always contained in the manifold. It is called standard 2 -chain $\mu_{0}$ (with respect to the given normalization). One can define analytic 2-chains as all possible images of the standard 2-chain under renormalizations. The family of chains passing through a given point does not depend on the choice of normal coordinates but it does depend on the initial point. In difference to the situation for hypersurfaces a 2-chain $\mu$ for the initial point $p$ need not be a 2-chain for other points $p^{\prime} \in \mu$. 
It is easy to obtain the analytic 2-chains for the quadrics through the origin as the images of the standard chain under isotropic automorphisms. Thus these 2-chains coincide with the geometrically defined chains from 2.4. One obtains that unparameterized 2-chains are the intersections of the quadric with so-called matrix lines $Z=A W$, where $Z, W, A$ have the same meaning as above. Automorphisms with $C=1, A=0$ preserve the standard chain and change only the parameter. Since the renormalizations of a manifold coincide up to higher order terms with automorphisms of the osculating quadric this shows that there exists exactly one 2-chain through the origin tangent to $\{Z=A U\}$.

The 1-chains considered in 2.4 can be easily described for quadrics (see [19]). The projections of the 1-parametric families from $\mathfrak{g}_{-2}$ are straight lines in $\mu_{0}$ through the origin. All other 1-chains are obtained by the action of isotropic automorphisms. Since the latter preserve 2-chains it follows that 1-chains always remain in some 2-chain. The isotropic automorphisms decompose into one automorphism that preserves $\mu_{0}$ and one that maps $\mu_{0}$ to another chain. Therefore it suffices to study the 1-chains that are contained in $\mu_{0}$. For the hyperbolic quadric we have the following situation:

- There are two singular directions at $\mu_{0}$ such that the only 1-chains in these directions are straight lines: $\left\{u_{1}=0\right\}$ and $\left\{u_{2}=0\right\}$.

- In all non-singular directions one has a 1-parametric family of 1-chains consisting of one straight line and hyperbolas

$$
u_{1}=\frac{\alpha u_{2}}{1-\beta u_{2}}
$$

where $\alpha$ indicates the direction and $\beta$ is the additional parameter.

- 2-chains may intersect at single points or singular 1-chains only.

In the elliptic case we have

- In any direction of $\mu_{0}$ there is a 1-parametric family of 1-chains consisting of a straight line and circles

$$
\beta\left(u_{1}^{2}+u_{2}^{2}\right)+\sin \alpha u_{1}-\cos \alpha u_{2}=0,
$$

where $\alpha$ indicates the direction and $\beta$ is the additional parameter.

- 2-chains intersect at single points.

\section{REFERENCES}

[1] T.N. BAiley, M.G. EAstwood, AND C.R. GRAHAM, Invariant theory for conformal and CR geometry, Annals of Mathematics, 139 (1994), pp. 491-552.

[2] R.J. Baston and M.G. Eastwood, The Penrose Transform, Its Interaction with Representation Theory, Clarenden press, Oxford, 1989.

[3] A. ČAP, Partially integrable almost CR-manifolds, Preprint, to appear.

[4] A. ĊAP AND H. Schichl, Parabolic geometries and canonical Cartan connections, Preprint ESI 450 , electronically available at www.esi.ac.at..

[5] A. ĊAP, J. SlOVÁK, AND V. SouČEK, Invariant Operators on Manifolds with Almost Hermitian Structures. I. Invariant Differentiation, Acta Math. Univ. Commenianae, 66:1 (1997), pp. 33-69; II. Normal Cartan Connections, Acta Math. Univ. Commenianae, 66:2 (1997), pp. 203-220 (Both electronically available at www.emis.de); III. Standard operators, Preprint ESI 613, electronically available at www.esi.ac.at, to appear in Diff. Geom. and its Appl..

[6] A. ČAP, J. SLOVÁK, AND V. SouČEK, Bernstein-Gelfand-Gelfand sequences,, ESI Preprint 722, electronically available at www.esi.ac.at..

[7] E. CARTAN, Sur la géométrie pseudo-conforme des hypersurfaces de deux variables complexes I., Ann. Math. Pures Apl., 11 (1932), pp. 17-90; II. Ann. Scuola Norm. Sup. Pisa, 1 (1932), pp. 333-354. 
[8] S.S. Chern AND J. J. Moser, Real hypersurfaces in complex manifolds, Acta Math., 133 (1974), pp. 219-271.

[9] V. V. Ezhov, A.V. IsAev, AND G. Schmalz, Invariants of elliptic and hyperbolic CR-structures of codimension 2, Int. J. Math, 10:1 (1999), pp. 1-52.

[10] V. V. Ezhov AND G. SchmalZ, Holomorphic automorphisms of quadrics, Math. Z., 216 (1994), pp. $453-470$.

[11] V. V. Ezhov AND G. Schmalz, Normal form and 2-dimensional chains of an elliptic CR surface in $\mathbb{C}^{4}$, Jour. Geom. Analysis, 6:4 (1996), pp. 495-529.

[12] C. Fefferman, Parabolic invariant theory in complex analysis, Adv. in Math., 31 (1979), pp. $131-262$.

[13] T. GARRITY AND R. MIZNER, The equivalence problem for higher co-dimensional CR structures, Pacific J. Math., 177 (1997), pp. 211-235.

[14] A. R. Gover, Aspects of parabolic invariant theory, in Proceedings Winter School Geometry and Physics, Srní, 1998, Supp. Rend. Circ. Matem. Palermo, Ser. II, 59 (1999), pp. 25-47.

[15] B. Kostant, Lie algebra cohomology and the generalized Borel-Weil theorem, Ann. Math., 74:2 (1961), pp. 329-387.

[16] A. V. LOBODA, Generic real analytic manifolds of codimension 2 in $\mathbb{C}^{4}$ and their biholomorphic mappings, Math. USSR Izvestiya, 33:2 (1989), pp. 295-315.

[17] R. MIZNER, CR-structures of codimension 2, J. Diff. Geom., 30:1 (1989), pp. 167-191.

[18] T. MоRiмото, Geometric structures on filtered manifolds, Hokkaido Math. J., 22 (1993), pp. 263-347.

[19] G. Schmalz, Remarks on CR-manifolds of codimension 2 in $\mathbb{C}^{4}$, in Proceedings Winter School Geometry and Physics, Srní, 1998, Supp. Rend. Circ. Matem. Palermo, Ser. II, 59 (1999), pp. 171-180.

[20] R. W. Sharpe, Differential Geometry (Cartan's Generalizations of Klein's Erlangen Program), Springer, GTM, New York, Berlin, 1997.

[21] J. Slovák, Parabolic Geometries, Part of the DrSc Dissertation, Preprint IGA 11/97, electronically available at www.maths.adelaide.edu.au..

[22] N. TANAKA, On the pseudo-conformal geometry of hypersurfaces of the space of $n$ complex variables, J. Math. Soc. Japan, 14 (1962), pp. 397-429.

[23] N. TANAKA, On non-degenerate real hypersurfaces, graded Lie algebras and Cartan connections, Japan. J. of Math., 2 (1976), pp. 131-190.

[24] N. TANAKA, On the equivalence problem associated with simple graded Lie algebras, Hokkaido Math. J., 8 (1979), pp. 23-84.

[25] D. A. Vogan, Representation of Real Reductive Lie Groups, Progress in Mathematics 15, Birkhäuser, Boston, 1981.

[26] S. M. WeBster, Pseudo-hermitian structures on a real hypersurface, J. Diff. Geom., 13 (1978), pp. $25-41$.

[27] K. YamAGUCHI, Differential systems associated with simple graded Lie algebras, Advanced Studies in Pure Mathematics, 22 (1993), pp. 413-494. 
